\title{
Source Energy and Emission Factors for Energy Use in Buildings
}

Technical Report NREL/TP-550-38617

Revised June 2007

M. Deru and P. Torcellini 


\section{Source Energy and Emission Factors for Energy Use in Buildings}

M. Deru and P. Torcellini

Prepared under Task No. BEC71004

Technical Report NREL/TP-550-38617

Revised June 2007

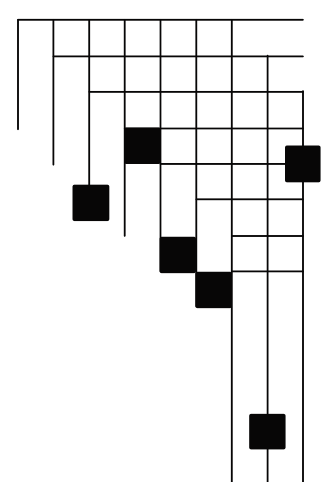




\section{NOTICE}

This report was prepared as an account of work sponsored by an agency of the United States government. Neither the United States government nor any agency thereof, nor any of their employees, makes any warranty, express or implied, or assumes any legal liability or responsibility for the accuracy, completeness, or usefulness of any information, apparatus, product, or process disclosed, or represents that its use would not infringe privately owned rights. Reference herein to any specific commercial product, process, or service by trade name, trademark, manufacturer, or otherwise does not necessarily constitute or imply its endorsement, recommendation, or favoring by the United States government or any agency thereof. The views and opinions of authors expressed herein do not necessarily state or reflect those of the United States government or any agency thereof.

Available electronically at http://www.osti.gov/bridge

Available for a processing fee to U.S. Department of Energy and its contractors, in paper, from:

U.S. Department of Energy

Office of Scientific and Technical Information

P.O. Box 62

Oak Ridge, TN 37831-0062

phone: 865.576 .8401

fax: 865.576 .5728

email: mailto:reports@adonis.osti.gov

Available for sale to the public, in paper, from:

U.S. Department of Commerce

National Technical Information Service

5285 Port Royal Road

Springfield, VA 22161

phone: 800.553.6847

fax: 703.605.6900

email: orders@ntis.fedworld.gov

online ordering: http://www.ntis.gov/ordering.htm 


\section{Source Energy and Emission Factors for Energy Use in Buildings}

\section{Errata}

The June 2006 version of NREL/TP-550-38617 contained errors in the emissions data that have been corrected with this revised version of the report. The wrong numbers were displayed for the emission factors for mercury, PM10, and solid waste. In addition to correcting the errors, revised calculation methods were used and subbituminous coal was treated separately from bituminous coal. In addition, the emissions for $\mathrm{CO}_{2}, \mathrm{SO}_{2}, \mathrm{NO}_{\mathrm{X}}$, and mercury from fossil fuels was adjusted to align with data from the EIA Electric Power Annual 2006 and EPA eGRID 2006. Finally, ozone season $\mathrm{NO}_{\mathrm{X}}$ emission factors by state are included at the end of the document.

The following specific changes were made to the document:

1. The emission factor name "Equivalent carbon dioxide $\left(\mathrm{CO}_{2 \mathrm{e}}\right)$ " replaces "global warming potential (GWP)." GWP is an index comparing the radiative properties of a compound to that of $\mathrm{CO}_{2}$.

2. The following tables were updated with revised energy and emission factors: 1 to 4, 6 to 11 , B-1 to B-11.

3. Figure 1 was replaced with the new figure from NERC with the new subregions.

4. Figure 2 was replaced with a graph of the revised data.

5. Table B-12 was added.

6. Two references were added with life cycle analysis data on the construction and operation of coal and natural gas fired power plants. 


\section{Acknowledgments}

The authors would like to thank the following individuals for reviewing this document: David Hansen, Drury Crawley, and John Ryan of DOE, Ron Judkoff, Craig Christensen, Dennis Barley, and Shanti Pless of NREL. In addition, the authors would like to thank James Littlefield of Franklin Associates for technical advice on energy and emissions inventories. 


\section{Contents}

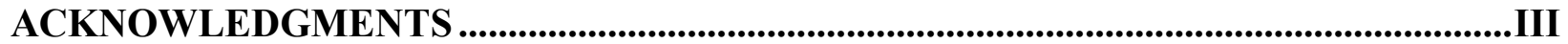

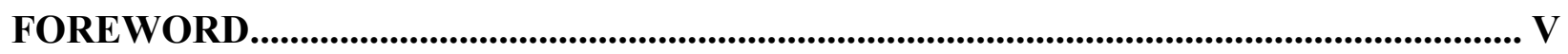

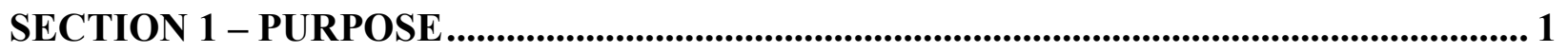

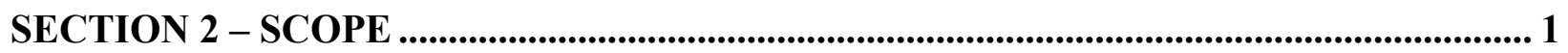

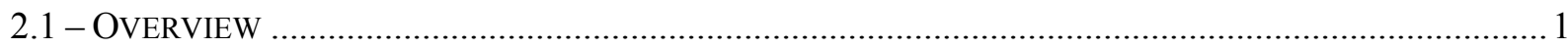

2.2 - WHAT THIS REPORT DOES .............................................................................................

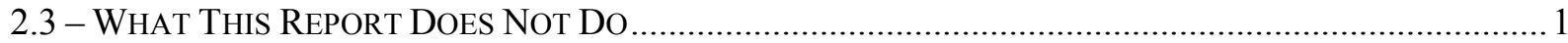

SECTION 3 - DEFINITIONS, ABBREVIATIONS, AND ACRONYMS ............................... 1

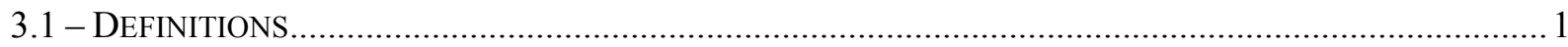

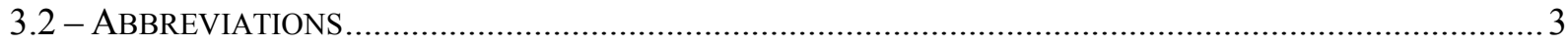

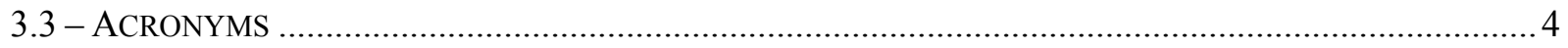

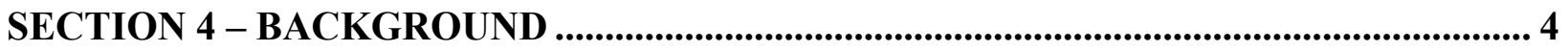

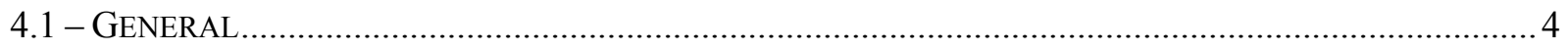

4.2 - ELECTRICITY GENERATION AND DISTRIBUTION ……………………………………………. 5

SECTION 5 - ENERGY AND EMISSION FACTORS....................................................... 7

5.1 - ELECTRICITY ENERGY AND EMISSION FACTORS …………………………………………...... 7

5.2 - DELIVERED FUEL ENERGY AND EMISSION FACTORS …………………………………………... 9

5.3 - EMISSION FACTORS FOR ON-SITE COMBUSTION ……………………………………….... 10

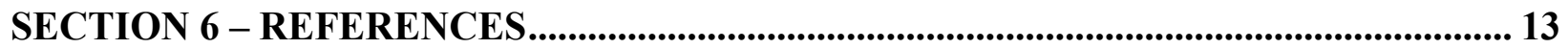

APPENDIX A - DERIVATION OF ENERGY AND EMISSION FACTORS........................ 14

APPENDIX B - ADDITIONAL ENERGY AND EMISSION DATA ................................... 19 


\section{Foreword}

People measure and analyze the energy performance of buildings for many reasons. Comparisons of energy use may be made between nations, regions, individual buildings, or systems within a building. Policy makers, owners, designers, operators, raters, and researchers use energy performance data. Many tools (or approaches) have been developed to analyze energy performance in different ways, at different levels of effort and precision, and at different stages in the life of a building. Each tool quantifies the building energy performance to fit the users' needs. However, methods and metrics are often inconsistent with each other. In addition, performance numbers may be misrepresented or misused to predict energy savings beyond the accuracy of the numbers.

The Performance Metrics Project is a U.S. Department of Energy commercial buildings research activity whose goal is to standardize the measurement and characterization of building energy performance. Its main products are clearly defined energy performance metrics and standard procedures for determining the performance metrics; its intents are to define common language and to create standards that produce consistent results independently of the user. Therefore, the terms and techniques are clearly defined with little room for interpretation. The more opportunity there is for interpretation, the higher the probability for disparity and "gaming" of the results. These procedures focus on reporting absolute numbers and not on comparisons of energy performance. Benchmarks are included only where well-established values apply. However, benchmarking of results by others can be improved by using the clearly defined absolute metrics determined by these procedures.

This document supports the other measurement procedures and all building energy-monitoring projects by providing methods to calculate the source energy and emissions from the energy measured at the building. Energy and emission factors typically account for the conversion inefficiencies at the power plant and the transmission and distribution losses from the power plant to the building. The energy and emission factors provided here also include the precombustion effects, which are the energy and emissions associated with extracting, processing, and delivering the primary fuels to the point of conversion in the electrical power plants or directly in the buildings. Source energy factors for electricity are provided for the total fossil fuel, total nonrenewable energy, and total energy. The breakdown of the energy used to generate electricity is provided on the national level, interconnection level, and state level. 


\section{Section 1 - Purpose}

This procedure provides source energy factors and emission factors to calculate the source (primary) energy and emissions from a building's annual site energy consumption.

\section{Section 2 - Scope}

\section{1 - Overview}

Source energy consumption and emissions from energy use must be calculated to determine the environmental impact of buildings; however, these calculations are often not completed and are usually not done in a consistent manner. Most analyses use the energy measured at the site, which is useful for understanding the performance of the building and the building systems, but it is not a good indicator of the environmental impacts from resource consumption and emissions associated with energy use. Site energy is also not a good metric for comparing buildings that use different energy types, buildings with on-site energy generation such as photovoltaics (PV), or buildings with cogeneration systems.

\section{2 - What This Report Does}

This report provides the energy and emission factors to calculate the source energy and emissions for electricity and fuels delivered to a facility and combustion of fuels at a facility. The factors for electricity are broken down by fuel type and presented for the continental United States, three grid interconnections, and each state. The electricity fuel and emission factors are adjusted for the electricity and the useful thermal output generated by combined heat and power (CHP) plants larger than one megawatt. The energy and emissions from extracting, processing, and transporting the fuels, also known as the precombustion effects, are included.

\section{3 - What This Report Does Not Do}

This report does not provide hourly or seasonal source energy factors. Time of use effects for electricity can be significant, but the data for such an analysis are difficult to obtain on a national basis and are beyond the scope of this report. The emissions from and the energy used for construction and decommissioning the power plants, hydroelectric dams, and energy infrastructure are not included. The energy and climate change emissions from these phases form a small fraction of the energy and emissions produced over the life of the facilities (Spath et al. 1999; Spath and Mann 2000). The energy associated with storage and disposal of spent nuclear waste is not included because reliable data are not available. This information should be analyzed when it becomes available to determine whether it is significant.

\section{Section 3 - Definitions, Abbreviations, and Acronyms}

\section{1 - Definitions}

Annual

A period that consists of 12 consecutive months.

\section{Criteria Pollutants}

The U.S. Environmental Protection Agency (EPA) has set national air quality standards for six principal air pollutants (also referred to as criteria pollutants): nitrogen dioxide $\left(\mathrm{NO}_{2}\right)$, ozone, sulfur dioxide $\left(\mathrm{SO}_{2}\right)$, particulate matter $(\mathrm{PM})$, carbon monoxide $(\mathrm{CO})$, and lead. Four of these pollutants $\left(\mathrm{CO}\right.$, lead, $\mathrm{NO}_{2}$, and $\left.\mathrm{SO}_{2}\right)$ result primarily from direct emissions from a variety of sources. PM results from direct emissions, but is also commonly formed when emissions of nitrogen oxides $\left(\mathrm{NO}_{\mathrm{x}}\right)$, sulfur oxides $\left(\mathrm{SO}_{\mathrm{x}}\right)$, ammonia, organic compounds, and other gases react in the atmosphere. Ozone is 
not directly emitted, but is formed when $\mathrm{NO}_{\mathrm{x}}$ and volatile organic compounds react in the presence of sunlight (EPA 2005a).

\section{Emission Factor}

The mass of a pollutant emitted to the environment per unit of energy or fuel associated with the production, distribution, and use of the energy or fuel.

Facility

A set of one or more buildings or outdoor applications, or both, that is defined for the purpose of an energy analysis. The boundary of the facility used in the energy analysis should be clearly defined.

Fuel Oil

A liquid petroleum product that includes distillate fuel oil (No. 1, No. 2, and No. 4), and residual fuel oil (No. 5 and No. 6).

Distillate Fuel Oil: A general classification for one of the petroleum fractions produced in conventional distillation operations. It is used primarily for space heating, on- and off-highway diesel engine fuel (including railroad engine fuel and fuel for agriculture machinery), and electric power generation. Included are Fuel Oils No. 1, No. 2, and No. 4; and Diesel Fuels No. 1, No. 2, and No. 4. Fuel oils and diesel fuel oils are very similar, but they do have different specifications.

Residual Fuel Oil: The topped crude of refinery operation that includes No. 5 and No. 6 fuel oils as defined in American Society for Testing and Materials Specification D396 and Federal Specification VV-F-815C; Navy Special fuel oil as defined in Military Specification MIL-F-859E, including Amendment 2 (NATO Symbol F-77); and Bunker C fuel oil. Residual fuel oil is used to produce electric power, space heating, vessel bunkering, and for various industrial purposes. Imports of residual fuel oil include imported crude oil burned as fuel (EIA 2005a).

\section{Global Warming Potential (GWP)}

GWP is an index that describes the radiative characteristics of well-mixed greenhouse gases. It represents the combined effect of the times these gases remain in the atmosphere and their relative effectiveness in absorbing outgoing infrared radiation. This index approximates the time-integrated warming effect of a unit mass of a given greenhouse gas in today's atmosphere, relative to that of carbon dioxide $\left(\mathrm{CO}_{2}\right)$. GWP is an index for estimating the relative global warming contribution of atmospheric emissions of $1 \mathrm{~kg}$ of a particular greenhouse gas compared to emissions of $1 \mathrm{~kg} \mathrm{of} \mathrm{CO}_{2}$. This document uses the following GWPs based on a 100-year time horizon: 1 for $\mathrm{CO}_{2}, 23$ for methane $\left(\mathrm{CH}_{4}\right)$, and 296 for nitrous oxide $\left(\mathrm{N}_{2} \mathrm{O}\right)$ (IPCC 2001). The equivalent $\mathrm{CO}_{2}$ emissions are calculated with the GWPs.

Measure

To determine a quantity with a calibrated instrument. This includes using previously measured data such as those shown on a utility bill or engineering log.

Metric

A standard definition of a measurable quantity.

Particulate Matter PM10 and PM2.5

Particulates can be directly introduced to the atmosphere from transportation vehicles, combustion, farming, and dirt roads. Particulates can also be formed in the atmosphere from chemical reactions of sunlight with emissions of $\mathrm{NO}_{\mathrm{x}}, \mathrm{SO}_{\mathrm{x}}$, ammonia, organic compounds, and other gases. PM10 is measure of particles in the atmosphere with a diameter of 10 micrometers or smaller. PM2.5 is a measure of particles 2.5 micrometers and smaller in the air and are sometimes called fine particles. PM2.5 have been associated with significant respiratory and cardiovascular health risks; however, 
consistent and reliable data for emission factors are not available. EPA maintains separate National Ambient Air Quality Standards for PM10 and PM2.5 because they have different monitoring and implementation plans (EPA 2005a).

\section{Performance Metric}

A standard definition of a measurable quantity that indicates some aspect of performance. Section 4 contains definitions of the specific performance metrics used in this procedure.

\section{Power Control Area (PCA)}

A PCA is a portion of an integrated power grid for which a single dispatcher has operational control of all electric generators. There are 112 PCAs reported in eGRID2006 (EPA 2007).

\section{Precombustion Effects}

The source energy used for and the emissions resulting from extracting, processing, and delivering a fuel to the point of use in a power plant or a building.

\section{Primary Energy}

The sum of the energy consumed at a facility and the energy required to extract, convert, and transmit that energy to the facility. (same as source energy)

\section{Site Energy}

The energy directly consumed at a facility typically measured with utility meters.

\section{Source Energy}

The sum of the energy consumed at a facility and the energy required to extract, convert, and transmit that energy to the facility. The source energy for electricity from hydroelectric power, solar energy, and wind is assumed to be equal to the electricity produced at the source; however, the transmission and distribution losses are accounted for in the electricity delivered to the facility. Source energy for electricity from thermal electric power plants fueled by geothermal and biomass is determined by assuming an efficiency of $33 \%$ for electricity production.

\section{Source Energy Factor}

The unit of source energy consumed per unit of energy or fuel delivered to the facility.

\section{Useful Thermal Output}

The thermal energy made available for use in any industrial or commercial process, or used in any heating or cooling application; i.e., total thermal energy made available for processes and applications other than electrical generation (EIA 2005b).

Year

A period of 365 consecutive days.

\section{2 - Abbreviations}

$\begin{array}{ll}\text { Anth } & \text { anthracite coal } \\ \mathrm{Bit} & \text { bituminous coal } \\ \mathrm{Btu} & \text { British thermal unit } \\ \mathrm{CH}_{4} & \text { methane } \\ \mathrm{CO} & \text { carbon monoxide } \\ \mathrm{CO}_{2} & \text { carbon dioxide } \\ \mathrm{CO}_{2 \mathrm{e}} & \text { equivalent carbon dioxide based on the GWP }\end{array}$




\begin{tabular}{|c|c|}
\hline GWP & global warming potential \\
\hline HPS & pumped hydro storage \\
\hline IP & inch-pound - refers to the English system of measurement units \\
\hline $\mathrm{kWh}$ & kilowatt hour \\
\hline LCI & life cycle inventory \\
\hline LPG & liquefied petroleum gas \\
\hline NO & nitrogen oxide \\
\hline $\mathrm{NO}_{\mathrm{x}}$ & nitrogen oxides \\
\hline $\mathrm{N}_{2} \mathrm{O}$ & nitrous oxide \\
\hline PCA & power control area \\
\hline PV & photovoltaic \\
\hline PM2.5 & particulate matter 2.5 micrometers in diameter and smaller \\
\hline PM10 & particulate matter 10 micrometers in diameter and smaller \\
\hline SI & International System of Units - refers to the metric system of measurement units \\
\hline $\mathrm{SO}_{\mathrm{x}}$ & sulfur oxides \\
\hline $\mathrm{SO}_{2}$ & sulfur dioxide \\
\hline Sub & subituminous coal \\
\hline $\mathrm{T} \& \mathrm{D}$ & transmission and distribution \\
\hline TNMOC & total non-methane organic compounds \\
\hline
\end{tabular}

\section{3 - Acronyms}

DOE U.S. Department of Energy

eGRID Emissions \& Generation Resource Integrated Database

EIA Energy Information Administration

EPA U.S. Environmental Protection Agency

ERCOT Electric Reliability Council of Texas

GREET Greenhouse Gases, Regulated Emissions, and Energy Use in Transportation

NERC North American Electrical Reliability Council

\section{Section 4 - Background}

\section{1 - General}

Most building energy analyses are completed with the energy measured on site. The building site energy is typically measured by the utility meters and is the total of the electrical, gas, and other energies delivered to the facility. Site energy consumption can be useful for understanding the performance of the building and the building systems, but it does not tell the whole story of the environmental impacts from resource consumption and emissions associated with the energy use. In addition, site energy is not a good comparison metric for buildings that have different mixes of energy types, buildings with on-site energy generation such as PV, or buildings with cogeneration units.

Energy analyses that use source or primary energy consumption typically use a fixed multiplier or source energy factor to estimate the source energy consumed to generate electricity based on national averages. There is usually no support or reference for the derivation of the source energy factor. For electricity production, the source energy multiplier typically varies from 3.0 to 3.33 depending on the study. This number is based on the assumption that most of the electricity was produced from thermal electric power plants. The result tells nothing of the fuel types consumed or the emissions from the electricity production. 


\section{2 - Electricity Generation and Distribution}

The electricity grid in the continental United States is divided into three main grids that have few connections and little energy transfer between them. These grids are named the Western Interconnection, Eastern Interconnection, and Electric Reliability Council of Texas (ERCOT) Interconnection (Figure 1). Note that the U.S., Canadian, and Mexican grids are separate with limited connections and energy transfer. Alaska and Hawaii have a variety of small local grids. The grid interconnections are managed by NERC through 10 regional reliability councils. The energy mixtures used to generate electricity in the continental United States, the three interconnections, Alaska, and Hawaii are listed in Table 1 and shown in Figure 2. For 2004 in the continental United States, the mix of primary energy used to generate electricity was $71 \%$ fossil fuels, $20 \%$ nuclear, $7 \%$ hydro, and $2 \%$ renewable. The "Other Fossil" fuel category includes tire-derived fuels, purchased steam, and other fuels.

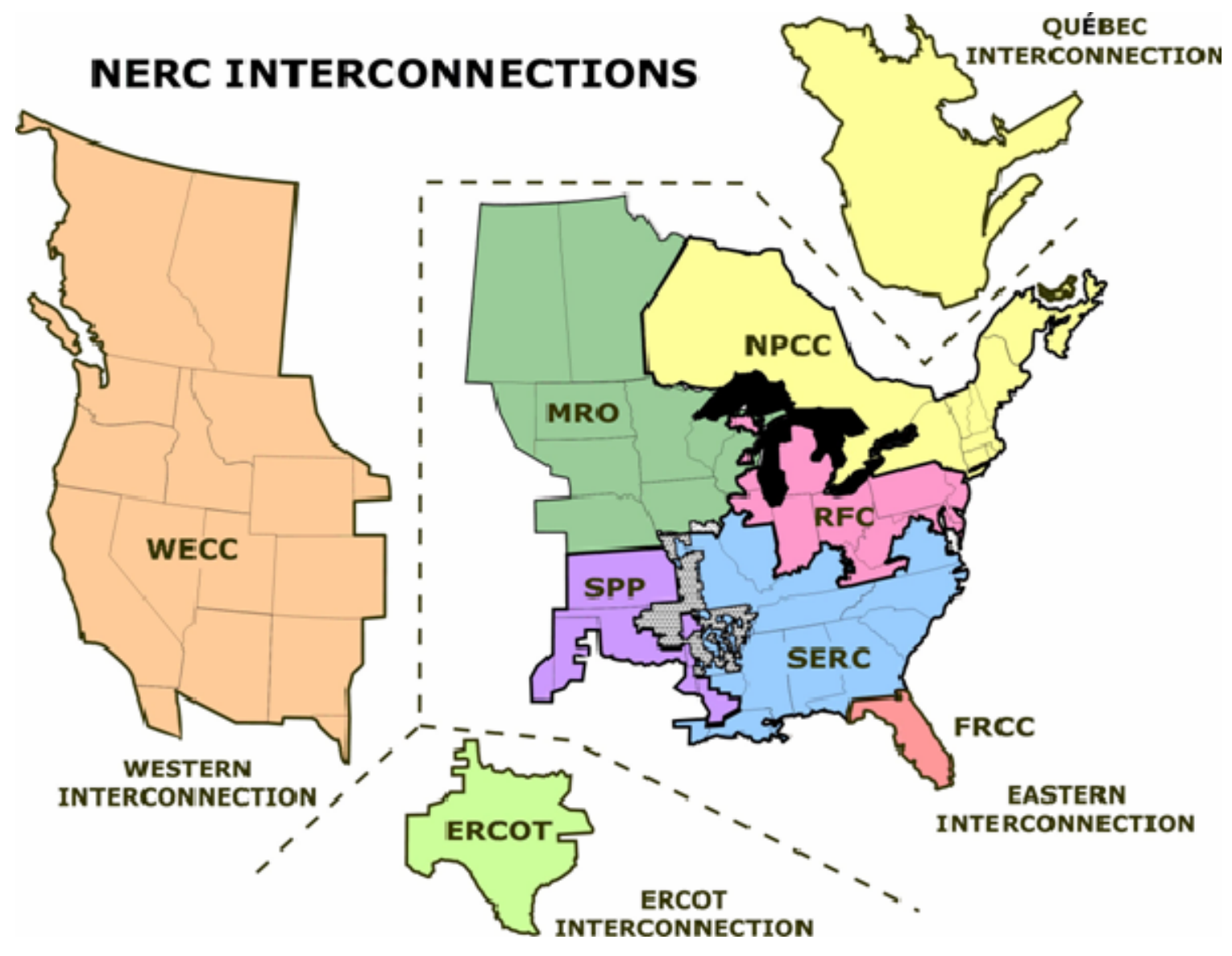

Figure 1 North American electrical grid interconnections, including the 10 NERC regional reliability councils (NERC 2007) 
Table 1 Percent Electricity Generation by Energy Type for 2004

\begin{tabular}{|l|r|r|r|r|r|r|}
\hline \multicolumn{1}{|c|}{ Energy Type } & $\begin{array}{c}\text { National } \\
\%\end{array}$ & $\begin{array}{c}\text { Eastern } \\
\%\end{array}$ & $\begin{array}{c}\text { Western } \\
\%\end{array}$ & $\begin{array}{c}\text { ERCOT } \\
\%\end{array}$ & $\begin{array}{c}\text { Alaska } \\
\%\end{array}$ & $\begin{array}{c}\text { Hawaii } \\
\%\end{array}$ \\
\hline Bituminous Coal & 27.8 & 34.3 & 13.1 & 0.0 & 0.0 & 1.0 \\
\hline Subbitumious Coal & 19.8 & 19.6 & 19.8 & 21.4 & 9.9 & 13.1 \\
\hline Lignite Coal & 2.3 & 1.4 & 0.0 & 14.8 & 0.0 & 0.0 \\
\hline Natural Gas & 18.3 & 12.7 & 27.4 & 49.4 & 55.5 & 1.5 \\
\hline Petroleum Fuels & 2.8 & 3.6 & 0.5 & 0.5 & 11.5 & 77.4 \\
\hline Other Fossil Fuel & 0.2 & 0.2 & 0.3 & 0.2 & 0.0 & 0.2 \\
\hline Nuclear & 19.9 & 23.0 & 9.9 & 12.4 & 0.0 & 0.0 \\
\hline Hydro & 6.8 & 3.4 & 24.6 & 0.3 & 23.0 & 0.8 \\
\hline Renewable Fuels & 1.5 & 1.7 & 1.3 & 0.2 & 0.1 & 4.2 \\
\hline Geothermal & 0.4 & 0.0 & 2.1 & 0.0 & 0.0 & 1.9 \\
\hline Wind & 0.4 & 0.1 & 1.0 & 0.9 & 0.0 & 0.1 \\
\hline Solar (PV) & 0.0 & 0.0 & 0.1 & 0.0 & 0.0 & 0.0 \\
\hline Fossil Fuel Total & 71.2 & 71.8 & 60.9 & 86.2 & 76.9 & 93.1 \\
\hline Renewable (non hydro) & 2.2 & 1.8 & 4.6 & 1.1 & 0.1 & 6.1 \\
\hline
\end{tabular}

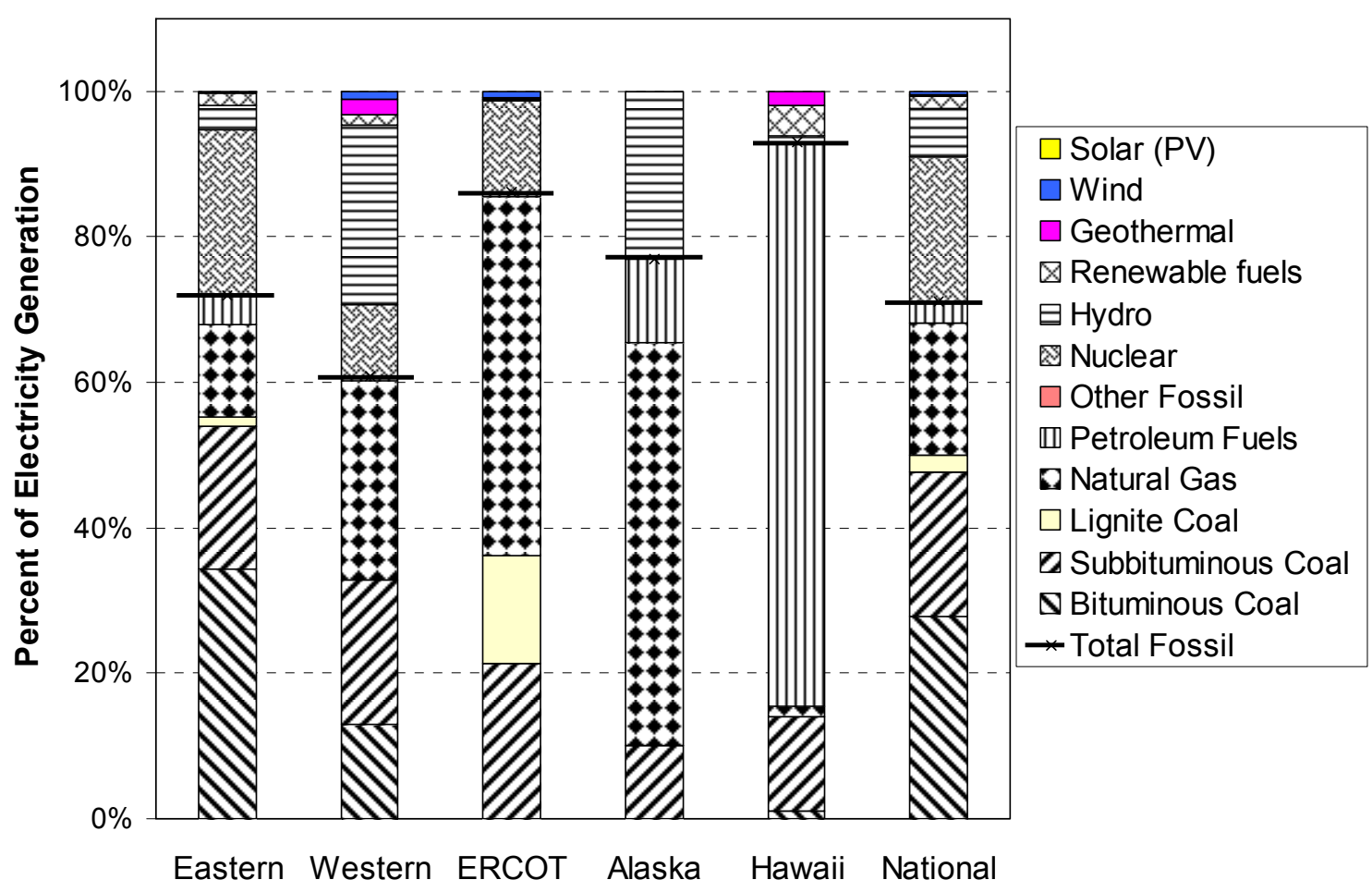

Figure 2 Electricity generation fuel mix for the continental United States (national), three interconnections, Alaska, and Hawaii for 2004 (EIA 2006a)

The question of what level of electricity generation data to use for a source energy analysis is not easy to answer. The options are national, interconnection, NERC region, NERC subregion, state, PCA, and utility. Typically, the goal of an energy analysis is to understand the impact of energy use or energy savings in a building compared to other options or to other buildings. It is often tempting to use source energy information at the utility level, which delivers the energy to the building. However, utilities are 
not isolated; energy is transferred around the grid where it is needed. Even between states, there is significant energy transfer; nine states in the Eastern Interconnection import more than $25 \%$ of their electricity. The District of Columbia imports more than $98 \%$ of its electricity. The source of the imported energy is difficult to account for. However, less than $1 \%$ of the energy generated and used with the interconnections is transferred to an adjacent interconnection. The interconnection level for fuel and emission factors within the continental United States should be used to ensure consistent analysis. The information for Alaska and Hawaii should be used for buildings in these states. One problem with using the interconnection level is that the boundaries of the interconnections are not well defined, and determining the correct interconnection for locations near the boundaries may be difficult. For example, some parts of Texas are outside of ERCOT; parts of northern and eastern Texas are in the Eastern Interconnection and parts of western Texas are in the Western Interconnection. The national fuel and emission factors should be used for cases where the interconnection is not known.

In cases where the analyzed building is within a state that has a small net imported amount of electricity or is a net exporter of electricity, the state-level data can be used. The net imports and exports of electricity by state are supplied in Table B-7 (Appendix B). Source energy and emission factors for electricity generation at the state level are listed in Tables B-9 and B-10.

\section{Section 5 - Energy and Emission factors}

\section{1 - Electricity Energy and Emission Factors}

Data from several sources were used to derive the energy and emission factors for electricity generation. (These data are summarized in Table A-1, and the calculation methods are presented in Appendix A. Addition data are presented in Appendix B.) The data presented are based on electricity generation in 2004 reported on EIA Forms 906 and 920 (EIA 2006a) and the U.S. Life Cycle Inventory (LCI) Database (NREL 2005).

Source energy factors per unit of delivered electricity are provided in Table 2. These factors include the transmission and distribution (T\&D) losses (first row of Table 2). Precombustion effects, which include extraction, processing, and transportation of the fuels, are also included. The precombustion energy accounts for $5 \%$ of the national total source energy factor and almost $12 \%$ of the total source factor for Hawaii. Energy to construct the power plants and energy infrastructures is not included as it is insignificant compared to the energy generated over the life of the facility. The Renewable Energy source energy factors are calculated using the fuel consumption reported on EIA forms 906 and 920 for geothermal and renewable fuels and assuming an energy factor of one for hydro, wind, and PV power plants. The T\&D losses are then added to get the source energy factor for the delivered electricity.

Table 2 Source Energy Factors for Delivered Electricity for 2004 ( $k W h$ of source energy per kWh of delivered electricity)

\begin{tabular}{|l|c|c|c|c|c|c|}
\hline & National & Eastern & Western & ERCOT & Alaska & Hawaii \\
\hline \hline T\&D Losses & $9.9 \%$ & $9.6 \%$ & $8.4 \%$ & $16.1 \%$ & $12.9 \%$ & $8.9 \%$ \\
\hline \hline Fossil Fuel Energy * & 2.500 & 2.528 & 2.074 & 3.168 & 3.368 & 3.611 \\
\hline Nonrenewable Energy ** & 3.188 & 3.321 & 2.415 & 3.630 & 3.386 & 3.653 \\
\hline Renewable Energy *** & 0.177 & 0.122 & 0.480 & 0.029 & 0.264 & 0.368 \\
\hline Total Energy & 3.365 & 3.443 & 2.894 & 3.658 & 3.650 & 4.022 \\
\hline
\end{tabular}

* Fossil Fuel Energy includes all coal, natural gas, petroleum fuels, and other fossil fuel

** Nonrenewable Energy includes Fossil Fuel Energy and nuclear

*** Renewable Energy includes hydro, renewable fuels, geothermal, wind, and solar PV 
The total emission factors (combustion plus precombustion) per delivered $\mathrm{kWh}$ of electricity for major pollutants in the continental United States and each major grid are shown in Tables 3 and 4. The separate emission factors from combustion and precombustion effects for electricity generation are shown in Tables B-3 through B-6 in IP and SI units. The precombustion emissions account for about $5 \%$ of the $\mathrm{CO}_{2}$ and $10 \%$ of the $\mathrm{CO}_{2 \mathrm{e}}$ emissions. The precombustion emissions of $\mathrm{CH}_{4}$ are approximately two orders of magnitude greater than the combustion emissions because of the high amount of $\mathrm{CH}_{4}$ released when coal is mined and processed.

The PM factors are for direct emissions and do not include the effect of particulate formation in the atmosphere from chemical reactions of sunlight with emissions of $\mathrm{NO}_{\mathrm{X}}, \mathrm{SO}_{\mathrm{X}}$, organic compounds, and other gases. The PM composition and emission levels are complex functions of boiler firing configuration, boiler operation, pollution control equipment, and fuel properties. The solid waste emissions consists of bottom ash, fly ash, boiler slag, and flue gas desulfurization sludge, minus the amount diverted from the waste stream and used in other products. The GWPs are based on 100-year time horizon for $\mathrm{CO}_{2}, \mathrm{CH}_{4}$, and $\mathrm{N}_{2} \mathrm{O}$. See the definition for GWP in Section 3.1 for more information.

Table 3 Total Emission Factors for Delivered Electricity (Ib of pollutant per kWh of electricity)

\begin{tabular}{|l|c|c|c|c|c|c|}
\hline \multicolumn{1}{|c|}{ Pollutant (Ib) } & National & Eastern & Western & ERCOT & Alaska & Hawaii \\
\hline \hline $\mathrm{CO}_{2 \mathrm{e}}$ & $1.67 \mathrm{E}+00$ & $1.74 \mathrm{E}+00$ & $1.31 \mathrm{E}+00$ & $1.84 \mathrm{E}+00$ & $1.71 \mathrm{E}+00$ & $1.91 \mathrm{E}+00$ \\
\hline $\mathrm{CO}_{2}$ & $1.57 \mathrm{E}+00$ & $1.64 \mathrm{E}+00$ & $1.22 \mathrm{E}+00$ & $1.71 \mathrm{E}+00$ & $1.55 \mathrm{E}+00$ & $1.83 \mathrm{E}+00$ \\
\hline $\mathrm{CH}_{4}$ & $3.71 \mathrm{E}-03$ & $3.59 \mathrm{E}-03$ & $3.51 \mathrm{E}-03$ & $5.30 \mathrm{E}-03$ & $6.28 \mathrm{E}-03$ & $2.96 \mathrm{E}-03$ \\
\hline $\mathrm{N}_{2} \mathrm{O}$ & $3.73 \mathrm{E}-05$ & $3.87 \mathrm{E}-05$ & $2.97 \mathrm{E}-05$ & $4.02 \mathrm{E}-05$ & $3.05 \mathrm{E}-05$ & $2.00 \mathrm{E}-05$ \\
\hline $\mathrm{NO} \times$ & $2.76 \mathrm{E}-03$ & $3.00 \mathrm{E}-03$ & $1.95 \mathrm{E}-03$ & $2.20 \mathrm{E}-03$ & $1.95 \mathrm{E}-03$ & $4.32 \mathrm{E}-03$ \\
\hline $\mathrm{SO} \times$ & $8.36 \mathrm{E}-03$ & $8.57 \mathrm{E}-03$ & $6.82 \mathrm{E}-03$ & $9.70 \mathrm{E}-03$ & $1.12 \mathrm{E}-02$ & $8.36 \mathrm{E}-03$ \\
\hline $\mathrm{CO}$ & $8.05 \mathrm{E}-04$ & $8.54 \mathrm{E}-04$ & $5.46 \mathrm{E}-04$ & $9.07 \mathrm{E}-04$ & $2.05 \mathrm{E}-03$ & $7.43 \mathrm{E}-03$ \\
\hline TNMOC & $7.13 \mathrm{E}-05$ & $7.26 \mathrm{E}-05$ & $6.45 \mathrm{E}-05$ & $7.44 \mathrm{E}-05$ & $8.40 \mathrm{E}-05$ & $1.15 \mathrm{E}-04$ \\
\hline Lead & $1.31 \mathrm{E}-07$ & $1.39 \mathrm{E}-07$ & $8.95 \mathrm{E}-08$ & $1.42 \mathrm{E}-07$ & $6.30 \mathrm{E}-08$ & $1.32 \mathrm{E}-07$ \\
\hline Mercury & $3.05 \mathrm{E}-08$ & $3.36 \mathrm{E}-08$ & $1.86 \mathrm{E}-08$ & $2.79 \mathrm{E}-08$ & $3.80 \mathrm{E}-08$ & $1.72 \mathrm{E}-07$ \\
\hline PM10 & $9.16 \mathrm{E}-05$ & $9.26 \mathrm{E}-05$ & $6.99 \mathrm{E}-05$ & $1.30 \mathrm{E}-04$ & $1.09 \mathrm{E}-04$ & $1.79 \mathrm{E}-04$ \\
\hline Solid Waste & $1.90 \mathrm{E}-01$ & $2.05 \mathrm{E}-01$ & $1.39 \mathrm{E}-01$ & $1.66 \mathrm{E}-01$ & $7.89 \mathrm{E}-02$ & $7.44 \mathrm{E}-02$ \\
\hline
\end{tabular}

Table 4 Total Emission Factors for Delivered Electricity (kg of pollutant per kWh of electricity)

\begin{tabular}{|l|c|c|c|c|c|c|}
\hline \multicolumn{1}{|c|}{ Pollutant (kg) } & National & Eastern & Western & ERCOT & Alaska & Hawaii \\
\hline \hline $\mathrm{CO}_{2 \mathrm{e}}$ & $7.58 \mathrm{E}-01$ & $7.88 \mathrm{E}-01$ & $5.94 \mathrm{E}-01$ & $8.34 \mathrm{E}-01$ & $7.74 \mathrm{E}-01$ & $8.65 \mathrm{E}-01$ \\
\hline $\mathrm{CO}_{2}$ & $7.14 \mathrm{E}-01$ & $7.45 \mathrm{E}-01$ & $5.54 \mathrm{E}-01$ & $7.74 \mathrm{E}-01$ & $7.05 \mathrm{E}-01$ & $8.32 \mathrm{E}-01$ \\
\hline $\mathrm{CH}_{4}$ & $1.68 \mathrm{E}-03$ & $1.63 \mathrm{E}-03$ & $1.59 \mathrm{E}-03$ & $2.40 \mathrm{E}-03$ & $2.85 \mathrm{E}-03$ & $1.34 \mathrm{E}-03$ \\
\hline $\mathrm{N}_{2} \mathrm{O}$ & $1.69 \mathrm{E}-05$ & $1.76 \mathrm{E}-05$ & $1.35 \mathrm{E}-05$ & $1.82 \mathrm{E}-05$ & $1.38 \mathrm{E}-05$ & $9.06 \mathrm{E}-06$ \\
\hline $\mathrm{NO} \mathrm{X}$ & $1.25 \mathrm{E}-03$ & $1.36 \mathrm{E}-03$ & $8.84 \mathrm{E}-04$ & $9.98 \mathrm{E}-04$ & $8.83 \mathrm{E}-04$ & $1.96 \mathrm{E}-03$ \\
\hline $\mathrm{SO} \mathrm{X}$ & $3.79 \mathrm{E}-03$ & $3.89 \mathrm{E}-03$ & $3.09 \mathrm{E}-03$ & $4.40 \mathrm{E}-03$ & $5.09 \mathrm{E}-03$ & $4.10 \mathrm{E}-03$ \\
\hline $\mathrm{CO}$ & $3.65 \mathrm{E}-04$ & $3.87 \mathrm{E}-04$ & $2.48 \mathrm{E}-04$ & $4.12 \mathrm{E}-04$ & $9.31 \mathrm{E}-04$ & $3.37 \mathrm{E}-03$ \\
\hline $\mathrm{TNMOC}$ & $3.24 \mathrm{E}-05$ & $3.29 \mathrm{E}-05$ & $2.93 \mathrm{E}-05$ & $3.38 \mathrm{E}-05$ & $3.81 \mathrm{E}-05$ & $5.20 \mathrm{E}-05$ \\
\hline Lead & $5.92 \mathrm{E}-08$ & $6.30 \mathrm{E}-08$ & $4.06 \mathrm{E}-08$ & $6.44 \mathrm{E}-08$ & $2.86 \mathrm{E}-08$ & $5.99 \mathrm{E}-08$ \\
\hline Mercury & $1.39 \mathrm{E}-08$ & $1.52 \mathrm{E}-08$ & $8.42 \mathrm{E}-09$ & $1.27 \mathrm{E}-08$ & $1.72 \mathrm{E}-08$ & $7.79 \mathrm{E}-08$ \\
\hline PM10 & $4.16 \mathrm{E}-05$ & $4.20 \mathrm{E}-05$ & $3.17 \mathrm{E}-05$ & $5.92 \mathrm{E}-05$ & $4.94 \mathrm{E}-05$ & $8.12 \mathrm{E}-05$ \\
\hline Solid Waste & $8.63 \mathrm{E}-02$ & $9.28 \mathrm{E}-02$ & $6.29 \mathrm{E}-02$ & $7.55 \mathrm{E}-02$ & $3.58 \mathrm{E}-02$ & $3.37 \mathrm{E}-02$ \\
\hline
\end{tabular}




\section{2 - Delivered Fuel Energy and Emission Factors}

The source energy factors for fuel delivered to buildings are listed in Table 5. These factors represent the energy required to extract, process, and deliver the fuel to the building per unit of energy in the fuel assuming the heating values listed in the table. The precombustion emission factors for delivering fuels to buildings are shown in Tables 6 and 7. These numbers are based on national averages and are derived from the Fuels and Energy Precombustion LCI data module (NREL 2005). These emission factors do not include emissions from use of the fuel in the building, which is reported in Section 5.3.

Table 5 Source Energy Factors for Fuel Delivered to Buildings

\begin{tabular}{|l|c|r|r|}
\hline \multicolumn{1}{|c|}{ Fuel } & Source Energy Factor & \multicolumn{2}{c|}{ Higher Heating Value } \\
\hline \hline Anthracite Coal & 1.029 & $12,700 \mathrm{Btu} / \mathrm{lb}$ & $29,539 \mathrm{~kJ} / \mathrm{kg}$ \\
\hline Bituminous Coal & 1.048 & $12,155 \mathrm{Btu} / \mathrm{lb}$ & $28,270 \mathrm{~kJ} / \mathrm{kg}$ \\
\hline Subbitumious Coal & 1.066 & $8,818 \mathrm{Btu} / \mathrm{lb}$ & $20,509 \mathrm{~kJ} / \mathrm{kg}$ \\
\hline Lignite Coal & 1.102 & $6,465 \mathrm{Btu} / \mathrm{lb}$ & $15,038 \mathrm{~kJ} / \mathrm{kg}$ \\
\hline Natural Gas & 1.092 & $1,010 \mathrm{Btu} / \mathrm{ft}^{3}$ & $37,631 \mathrm{~kJ} / \mathrm{m}^{3}$ \\
\hline Residual Fuel Oil & 1.191 & $149,500 \mathrm{Btu} / \mathrm{gal}$ & $41,666 \mathrm{~kJ} / \mathrm{L}$ \\
\hline Distillate Fuel Oil & 1.158 & $138,700 \mathrm{Btu} / \mathrm{gal}$ & $38,656 \mathrm{~kJ} / \mathrm{L}$ \\
\hline Gasoline & 1.187 & $100,000 \mathrm{Btu} / \mathrm{gal}$ & $27,870 \mathrm{~kJ} / \mathrm{L}$ \\
\hline LPG & 1.151 & $91,000 \mathrm{Btu} / \mathrm{gal}$ & $25,362 \mathrm{~kJ} / \mathrm{L}$ \\
\hline Kerosene & 1.205 & $135,000 \mathrm{Btu} / \mathrm{gal}$ & $27,870 \mathrm{~kJ} / \mathrm{L}$ \\
\hline
\end{tabular}

* Heating value for $60^{\circ} \mathrm{F}$ and $14.70 \mathrm{psia}\left(15.6^{\circ} \mathrm{C}\right.$ and $\left.101325 \mathrm{~Pa}\right)$.

Table 6 Precombustion Emission Factors for Fuel Delivered to Buildings (lb of pollutant per unit of fuel)

\begin{tabular}{|c|c|c|c|c|c|c|c|c|c|}
\hline \multirow{2}{*}{$\begin{array}{l}\text { Pollutant } \\
\text { (Ib) }\end{array}$} & $\begin{array}{c}\text { Anthracite } \\
\text { Coal }\end{array}$ & $\begin{array}{c}\text { Bituminous } \\
\text { Coal }\end{array}$ & $\begin{array}{l}\text { Lignite } \\
\text { Coal }\end{array}$ & $\begin{array}{c}\text { Natural } \\
\text { Gas }\end{array}$ & $\begin{array}{l}\text { Residual } \\
\text { Fuel Oil }\end{array}$ & $\begin{array}{l}\text { Distillate } \\
\text { Fuel Oil }\end{array}$ & Gasoline & LPG & Kerosene \\
\hline & $1000 \mathrm{lb}$ & $1000 \mathrm{lb}$ & $1000 \mathrm{lb}$ & $1000 \mathrm{ft}^{3}$ * & $1000 \mathrm{gal}$ & $1000 \mathrm{gal}$ & $1000 \mathrm{gal}$ & $1000 \mathrm{gal}$ & $1000 \mathrm{gal}$ \\
\hline $\mathrm{CO}_{2 \mathrm{e}}$ & $9.76 \mathrm{E}+1$ & $1.89 \mathrm{E}+2$ & 1.37E+2 & $2.78 \mathrm{E}+1$ & $4.47 \mathrm{E}+3$ & $4.10 \mathrm{E}+3$ & $3.50 \mathrm{E}+3$ & $2.56 \mathrm{E}+3$ & $3.83 \mathrm{E}+3$ \\
\hline $\mathrm{CO}_{2}$ & $5.85 \mathrm{E}+1$ & $9.32 \mathrm{E}+1$ & $1.07 \mathrm{E}+2$ & $1.16 \mathrm{E}+1$ & $3.57 E+3$ & $3.28 \mathrm{E}+3$ & $2.80 \mathrm{E}+3$ & $2.05 \mathrm{E}+3$ & $3.06 \mathrm{E}+3$ \\
\hline $\mathrm{CH}_{4}$ & $1.69 \mathrm{E}+0$ & $4.15 \mathrm{E}+0$ & $1.30 \mathrm{E}+0$ & 7.04E-1 & $3.81 \mathrm{E}+1$ & $3.49 \mathrm{E}+1$ & $2.98 \mathrm{E}+1$ & $2.18 \mathrm{E}+1$ & $3.26 \mathrm{E}+1$ \\
\hline $\mathrm{N}_{2} \mathrm{O}$ & $1.08 \mathrm{E}-3$ & $1.80 \mathrm{E}-3$ & $1.45 \mathrm{E}-3$ & $2.35 \mathrm{E}-4$ & $6.57 \mathrm{E}-2$ & $6.03 \mathrm{E}-2$ & $5.14 \mathrm{E}-2$ & 3.77E-2 & $5.63 \mathrm{E}-2$ \\
\hline $\mathrm{NO}_{\mathrm{x}}$ & $2.51 \mathrm{E}-1$ & $7.69 \mathrm{E}-1$ & 3.33E-1 & $1.64 \mathrm{E}-2$ & $2.73 \mathrm{E}+1$ & $2.50 \mathrm{E}+1$ & $2.13 E+1$ & $1.57 \mathrm{E}+1$ & $2.34 \mathrm{E}+1$ \\
\hline $\mathrm{SO}_{\mathrm{x}}$ & $2.02 \mathrm{E}-1$ & $3.34 \mathrm{E}-1$ & $4.52 \mathrm{E}-1$ & $1.22 \mathrm{E}+0$ & $3.86 \mathrm{E}+1$ & $3.55 \mathrm{E}+1$ & $3.02 \mathrm{E}+1$ & $2.22 \mathrm{E}+1$ & $3.31 \mathrm{E}+1$ \\
\hline $\mathrm{CO}$ & $2.40 \mathrm{E}-1$ & $4.30 \mathrm{E}-1$ & 4.73E-1 & 1.36E-2 & $1.15 \mathrm{E}+2$ & $1.06 \mathrm{E}+2$ & $9.00 \mathrm{E}+1$ & $6.61 \mathrm{E}+1$ & $9.86 \mathrm{E}+1$ \\
\hline TNMOC & $3.74 \mathrm{E}-4$ & $7.36 \mathrm{E}-4$ & $8.55 \mathrm{E}-4$ & $4.56 \mathrm{E}-5$ & $2.31 \mathrm{E}-2$ & $2.12 \mathrm{E}-2$ & $1.81 \mathrm{E}-2$ & 1.33E-2 & $1.98 \mathrm{E}-2$ \\
\hline Lead & $3.44 \mathrm{E}-6$ & $5.21 \mathrm{E}-6$ & $3.13 \mathrm{E}-5$ & $2.41 \mathrm{E}-7$ & 1.47E-4 & 1.35E-4 & 1.15E-4 & 8.43E-5 & $1.26 \mathrm{E}-4$ \\
\hline Mercur & $7.45 \mathrm{E}-7$ & $1.29 \mathrm{E}-6$ & $1.20 \mathrm{E}-6$ & $5.51 \mathrm{E}-8$ & $2.42 \mathrm{E}-5$ & $2.22 \mathrm{E}-5$ & $1.89 \mathrm{E}-5$ & $1.39 \mathrm{E}-5$ & $2.07 \mathrm{E}-5$ \\
\hline PM10 & $6.04 \mathrm{E}-3$ & 2.10E-2 & $1.01 \mathrm{E}-2$ & $8.17 \mathrm{E}-4$ & $6.99 \mathrm{E}-1$ & $6.42 \mathrm{E}-1$ & $5.47 \mathrm{E}-1$ & $4.01 \mathrm{E}-1$ & $5.99 \mathrm{E}-1$ \\
\hline $\begin{array}{l}\text { PM- } \\
\text { unspecified }\end{array}$ & $2.11 \mathrm{E}+0$ & $1.65 \mathrm{E}+0$ & $1.31 \mathrm{E}-1$ & 1.42E-3 & $2.71 \mathrm{E}+0$ & $2.49 \mathrm{E}+0$ & $2.12 \mathrm{E}+0$ & $1.56 \mathrm{E}+0$ & $2.32 \mathrm{E}+0$ \\
\hline Solid Waste & $2.74 \mathrm{E}+2$ & $2.40 \mathrm{E}+2$ & $5.77 \mathrm{E}+0$ & $1.60 \mathrm{E}+0$ & $4.21 \mathrm{E}+2$ & $3.87 \mathrm{E}+2$ & $3.30 \mathrm{E}+2$ & $2.42 \mathrm{E}+2$ & $3.61 \mathrm{E}+2$ \\
\hline
\end{tabular}

* Gas volume at $60^{\circ} \mathrm{F}$ and 14.70 psia. 
Table 7 Precombustion Emission Factors for Fuel Delivered to Buildings

( $k g$ of pollutant per unit of fuel)

\begin{tabular}{|c|c|c|c|c|c|c|c|c|c|}
\hline \multirow{2}{*}{$\begin{array}{l}\text { Pollutant } \\
\text { (kg) }\end{array}$} & $\begin{array}{c}\text { Anthracite } \\
\text { Coal }\end{array}$ & $\begin{array}{c}\text { Bituminous } \\
\text { Coal }\end{array}$ & $\begin{array}{l}\text { Lignite } \\
\text { Coal }\end{array}$ & $\begin{array}{c}\text { Natural } \\
\text { Gas }\end{array}$ & $\begin{array}{l}\text { Residual } \\
\text { Fuel Oil }\end{array}$ & $\begin{array}{l}\text { Distillate } \\
\text { Fuel Oil }\end{array}$ & Gasoline & LPG & Kerosene \\
\hline & $1000 \mathrm{~kg}$ & 1000 kg & 1000 kg & $1000 \mathrm{~m}^{3}$ * & $1000 \mathrm{~L}$ & $1000 \mathrm{~L}$ & $1000 \mathrm{~L}$ & $1000 \mathrm{~L}$ & $1000 \mathrm{~L}$ \\
\hline $\mathrm{CO}_{2 \mathrm{e}}$ & $9.76 \mathrm{E}+1$ & $1.89 \mathrm{E}+2$ & $1.37 \mathrm{E}+2$ & 4.46E-1 & $5.35 \mathrm{E}+2$ & $4.92 \mathrm{E}+2$ & $4.19 \mathrm{E}+2$ & $3.07 \mathrm{E}+2$ & $4.59 \mathrm{E}+2$ \\
\hline $\mathrm{CO}_{2}$ & $5.85 \mathrm{E}+1$ & $9.32 \mathrm{E}+1$ & $1.07 \mathrm{E}+2$ & $1.86 \mathrm{E}-1$ & $4.28 \mathrm{E}+2$ & $3.93 \mathrm{E}+2$ & $3.35 \mathrm{E}+2$ & $2.46 \mathrm{E}+2$ & $3.67 \mathrm{E}+2$ \\
\hline $\mathrm{CH}_{4}$ & $1.69 \mathrm{E}+0$ & $4.15 \mathrm{E}+0$ & $1.30 \mathrm{E}+0$ & $1.13 \mathrm{E}-2$ & $4.56 \mathrm{E}+0$ & $4.19 \mathrm{E}+0$ & $3.57 \mathrm{E}+0$ & $2.62 \mathrm{E}+0$ & $3.91 \mathrm{E}+0$ \\
\hline $\mathrm{N}_{2} \mathrm{O}$ & $1.08 \mathrm{E}-3$ & $1.80 \mathrm{E}-3$ & $1.45 \mathrm{E}-3$ & $3.77 \mathrm{E}-6$ & $7.87 \mathrm{E}-3$ & $7.23 \mathrm{E}-3$ & $6.16 \mathrm{E}-3$ & $4.52 \mathrm{E}-3$ & $6.74 \mathrm{E}-3$ \\
\hline $\mathrm{NO}_{x}$ & $2.51 \mathrm{E}-1$ & $7.69 \mathrm{E}-1$ & $3.33 \mathrm{E}-1$ & $2.62 \mathrm{E}-4$ & $3.27 \mathrm{E}+0$ & $3.00 \mathrm{E}+0$ & $2.56 \mathrm{E}+0$ & $1.88 \mathrm{E}+0$ & $2.80 \mathrm{E}+0$ \\
\hline $\mathrm{SO}_{\mathrm{x}}$ & $2.02 \mathrm{E}-1$ & $3.34 \mathrm{E}-1$ & $4.52 \mathrm{E}-1$ & $1.95 \mathrm{E}-2$ & $4.63 \mathrm{E}+0$ & $4.25 \mathrm{E}+0$ & $3.62 \mathrm{E}+0$ & $2.66 \mathrm{E}+0$ & $3.96 \mathrm{E}+0$ \\
\hline $\mathrm{CO}$ & $2.40 \mathrm{E}-1$ & $4.30 \mathrm{E}-1$ & $4.73 \mathrm{E}-1$ & $2.18 \mathrm{E}-4$ & $1.38 \mathrm{E}+1$ & $1.27 \mathrm{E}+1$ & $1.08 \mathrm{E}+1$ & $7.91 \mathrm{E}+0$ & $1.18 \mathrm{E}+1$ \\
\hline TNMOC & $3.74 \mathrm{E}-4$ & $7.36 \mathrm{E}-4$ & $8.55 \mathrm{E}-4$ & $7.30 \mathrm{E}-7$ & $2.77 \mathrm{E}-3$ & $2.54 \mathrm{E}-3$ & $2.17 \mathrm{E}-3$ & $1.59 \mathrm{E}-3$ & $2.37 \mathrm{E}-3$ \\
\hline Lead & $3.44 \mathrm{E}-6$ & $5.21 \mathrm{E}-6$ & $3.13 \mathrm{E}-5$ & $3.86 \mathrm{E}-9$ & $1.76 \mathrm{E}-5$ & $1.62 \mathrm{E}-5$ & $1.38 \mathrm{E}-5$ & $1.01 \mathrm{E}-5$ & $1.51 \mathrm{E}-5$ \\
\hline Mercury & $7.45 \mathrm{E}-7$ & $1.29 \mathrm{E}-6$ & $1.20 \mathrm{E}-6$ & $8.82 \mathrm{E}-10$ & $2.89 \mathrm{E}-6$ & $2.66 \mathrm{E}-6$ & $2.26 \mathrm{E}-6$ & $1.66 \mathrm{E}-6$ & $2.48 \mathrm{E}-6$ \\
\hline PM10 & $6.04 \mathrm{E}-3$ & $2.10 \mathrm{E}-2$ & $1.01 \mathrm{E}-2$ & $1.31 \mathrm{E}-5$ & $8.38 \mathrm{E}-2$ & $7.70 \mathrm{E}-2$ & $6.56 \mathrm{E}-2$ & $4.81 \mathrm{E}-2$ & $7.18 \mathrm{E}-2$ \\
\hline $\begin{array}{l}\text { PM- } \\
\text { unspecified }\end{array}$ & $2.11 \mathrm{E}+0$ & $1.65 \mathrm{E}+0$ & $1.31 \mathrm{E}-1$ & $2.27 \mathrm{E}-5$ & $3.25 \mathrm{E}-1$ & $2.98 \mathrm{E}-1$ & $2.54 \mathrm{E}-1$ & $1.86 \mathrm{E}-1$ & $2.78 \mathrm{E}-1$ \\
\hline Solid Waste & $2.74 \mathrm{E}+2$ & $2.40 \mathrm{E}+2$ & $5.77 \mathrm{E}+0$ & $2.57 \mathrm{E}-2$ & $5.05 \mathrm{E}+1$ & $4.64 \mathrm{E}+1$ & $3.95 \mathrm{E}+1$ & $2.90 \mathrm{E}+1$ & $4.32 \mathrm{E}+1$ \\
\hline
\end{tabular}

* Gas volume at $15.6^{\circ} \mathrm{C}$ and $101325 \mathrm{~Pa}$.

\section{3 - Emission Factors for On-Site Combustion}

The emission factors for combustion of fuels in buildings are listed in Tables 8 to 11 . Unless otherwise noted, these emission factors are based on data from GREET 1.6 (ANL 2005), which uses EPA AP-42 (EPA 2005B) and other sources. The numbers include the emissions from combustion on site; the precombustion emissions in Tables 6 and 7 are not included. No data are available for some pollutants; this does not mean that the emissions are zero or negligible. The emission factors should be calculated for these pollutants when reliable data are available.

Emissions from combustion depend on the composition of the fuel, the equipment, and the maintenance of the equipment. In general, these factors provide baseline emissions from uncontrolled combustion sources. If more detailed information is known about the fuel or the combustion equipment, it should be used. 
Table 8 Emission Factors for On-Site Combustion in a Commercial Boiler (lb of pollutant per unit of fuel)

\begin{tabular}{|c|c|c|c|c|c|c|}
\hline \multirow{3}{*}{$\begin{array}{l}\text { Pollutant } \\
\text { (Ib) }\end{array}$} & \multicolumn{6}{|c|}{ Commercial Boiler } \\
\hline & $\begin{array}{c}\text { Bituminous } \\
\text { Coal * }\end{array}$ & $\begin{array}{l}\text { Lignite } \\
\text { Coal ** }\end{array}$ & Natural Gas & $\begin{array}{l}\text { Residual } \\
\text { Fuel Oil }\end{array}$ & $\begin{array}{c}\text { Distillate } \\
\text { Fuel Oil }\end{array}$ & LPG \\
\hline & $1000 \mathrm{lb}$ & $1000 \mathrm{lb}$ & $1000 \mathrm{ft}^{3 * * *}$ & $1000 \mathrm{gal}$ & $1000 \mathrm{gal}$ & 1000 gal \\
\hline$\overline{\mathrm{CO}_{2 \mathrm{e}}}$ & $2.74 \mathrm{E}+03$ & $2.30 \mathrm{E}+03$ & $1.23 E+02$ & $2.56 \mathrm{E}+04$ & $2.28 \mathrm{E}+04$ & $\overline{c 1.35 E+04}$ \\
\hline $\mathrm{CO}_{2}$ & $2.63 E+03$ & $2.30 \mathrm{E}+03$ & $1.22 \mathrm{E}+02$ & $2.55 E+04$ & $2.28 \mathrm{E}+04$ & $1.32 E+04$ \\
\hline $\mathrm{CH}_{4}$ & $1.15 \mathrm{E}-01$ & 2.00E-02 & $2.50 \mathrm{E}-03$ & $2.31 \mathrm{E}-01$ & 2.32E-01 & $2.17 \mathrm{E}-01$ \\
\hline $\mathrm{N}_{2} \mathrm{O}$ & $3.68 \mathrm{E}-01$ & $\mathrm{ND}^{\dagger}$ & $2.50 \mathrm{E}-03$ & $1.18 \mathrm{E}-01$ & $1.19 \mathrm{E}-01$ & $9.77 \mathrm{E}-01$ \\
\hline $\mathrm{NO}_{x}$ & $5.75 \mathrm{E}+00$ & $5.97 \mathrm{E}+00$ & $1.11 \mathrm{E}-01$ & $6.41 \mathrm{E}+00$ & $2.15 \mathrm{E}+01$ & $1.57 E+01$ \\
\hline $\mathrm{SO}_{\mathrm{x}}$ & $1.66 \mathrm{E}+00$ & $1.29 E+01$ & $6.32 \mathrm{E}-04$ & $4.00 \mathrm{E}+01$ & $3.41 \mathrm{E}+01$ & $0.00 E+00$ \\
\hline $\mathrm{CO}$ & $2.89 \mathrm{E}+00$ & $4.05 \mathrm{E}-03$ & 9.33E-02 & $5.34 \mathrm{E}+00$ & $5.41 \mathrm{E}+00$ & $2.17 E+00$ \\
\hline VOC & $\mathrm{ND}^{\dagger}$ & $\mathrm{ND}^{\dagger}$ & $6.13 \mathrm{E}-03$ & $3.63 E-01$ & $2.17 \mathrm{E}-01$ & $3.80 \mathrm{E}-01$ \\
\hline Lead & 1.79E-03 & 6.86E-02 & $5.00 \mathrm{E}-07$ & $1.51 \mathrm{E}-06$ & $\mathrm{ND}^{\dagger}$ & $\mathrm{ND}^{\dagger}$ \\
\hline Mercury & $6.54 \mathrm{E}-04$ & 6.54E-04 & $2.60 \mathrm{E}-07$ & 1.13E-07 & $\mathrm{ND}^{\dagger}$ & $\mathrm{ND}^{\dagger}$ \\
\hline PM10 & $2.00 \mathrm{E}+00$ & $\mathrm{ND}^{\dagger}$ & $8.40 \mathrm{E}-03$ & $4.64 \mathrm{E}+00$ & $1.88 \mathrm{E}+00$ & 4.89E-01 \\
\hline
\end{tabular}

Table 9 Emission Factors for On-Site Combustion in a Commercial Boiler (kg of pollutant per unit of fuel)

\begin{tabular}{|c|c|c|c|c|c|c|}
\hline \multirow{3}{*}{$\begin{array}{l}\text { Pollutant } \\
\text { (kg) }\end{array}$} & \multicolumn{6}{|c|}{ Commercial Boiler } \\
\hline & $\begin{array}{l}\text { Bituminous } \\
\text { Coal }^{*}\end{array}$ & $\begin{array}{l}\text { Lignite } \\
\text { Coal }\end{array}$ & Natural Gas & $\begin{array}{l}\text { Residual } \\
\text { Fuel Oil }\end{array}$ & $\begin{array}{c}\text { Distillate } \\
\text { Fuel Oil }\end{array}$ & LPG \\
\hline & $1000 \mathrm{~kg}$ & $1000 \mathrm{~kg}$ & $1000 \mathrm{~m}^{3^{* * *}}$ & $1000 \mathrm{~L}$ & $1000 \mathrm{~L}$ & $1000 \mathrm{~L}$ \\
\hline $\mathrm{CO}_{2 \mathrm{e}}$ & $2.74 \mathrm{E}+03$ & $2.30 \mathrm{E}+03$ & $1.97 \mathrm{E}+00$ & $3.06 \mathrm{E}+03$ & $2.73 E+03$ & $1.62 E+03$ \\
\hline $\mathrm{CO}_{2}$ & $2.63 \mathrm{E}+03$ & $2.30 \mathrm{E}+03$ & $1.96 \mathrm{E}+00$ & $3.06 \mathrm{E}+03$ & $2.73 E+03$ & $1.59 E+03$ \\
\hline $\mathrm{CH}_{4}$ & 1.15E-01 & 2.00E-02 & 4.00E-05 & 2.76E-02 & $2.78 \mathrm{E}-02$ & 2.60E-02 \\
\hline $\mathrm{N}_{2} \mathrm{O}$ & 3.68E-01 & $\mathrm{ND}^{\dagger}$ & 4.00E-05 & $1.41 \mathrm{E}-02$ & 1.43E-02 & 1.17E-01 \\
\hline $\mathrm{NO}_{\mathrm{x}}$ & $5.75 \mathrm{E}+00$ & $5.97 \mathrm{E}+00$ & 1.78E-03 & 7.68E-01 & $2.58 \mathrm{E}+00$ & $1.88 \mathrm{E}+00$ \\
\hline $\mathrm{SO}_{\mathrm{X}}$ & $1.66 \mathrm{E}+00$ & $1.29 \mathrm{E}+01$ & 1.01E-05 & $4.79 \mathrm{E}+00$ & $4.09 \mathrm{E}+00$ & $0.00 E+00$ \\
\hline $\mathrm{CO}$ & $2.89 \mathrm{E}+00$ & 4.05E-03 & 1.50E-03 & $6.40 \mathrm{E}-01$ & 6.48E-01 & 2.60E-01 \\
\hline VOC & $\mathrm{ND}^{\dagger}$ & $\mathrm{ND}^{\dagger}$ & 9.82E-05 & 4.35E-02 & 4.39E-02 & 4.55E-02 \\
\hline Lead & 1.79E-03 & $6.86 \mathrm{E}-02$ & 8.01E-09 & $1.81 \mathrm{E}-07$ & $\mathrm{ND}^{\dagger}$ & $\mathrm{ND}^{\dagger}$ \\
\hline Mercury & $6.54 \mathrm{E}-04$ & $6.54 \mathrm{E}-04$ & 4.16E-09 & $1.35 \mathrm{E}-08$ & $\mathrm{ND}^{\dagger}$ & $\mathrm{ND}^{\dagger}$ \\
\hline PM10 & $2.00 \mathrm{E}+00$ & $\mathrm{ND}^{\dagger}$ & 1.35E-04 & 5.56E-01 & $2.25 \mathrm{E}-01$ & 5.86E-02 \\
\hline
\end{tabular}

* from the U.S. LCI data module: Bituminous Coal Combustion in an Industrial Boiler (NREL 2005)

** from the U.S. LCI data module: Lignite Coal Combustion in an Industrial Boiler (NREL 2005)

*** Gas volume at $15.6^{\circ} \mathrm{C}$ and $101325 \mathrm{~Pa}$.

${ }^{\dagger}$ no data available 
Table 10 Emission Factors for On-Site Combustion in Other Equipment (Ib of pollutant per unit of fuel)

\begin{tabular}{|c|c|c|c|c|c|c|}
\hline \multirow{3}{*}{$\begin{array}{l}\text { Pollutant } \\
\text { (Ib) }\end{array}$} & \multicolumn{3}{|c|}{ Stationary Reciprocating Engine } & \multicolumn{2}{|c|}{ Small Turbine } & \multirow{2}{*}{$\begin{array}{c}\begin{array}{c}\text { Residential } \\
\text { Furnace * }\end{array} \\
\text { Natural Gas }\end{array}$} \\
\hline & Natural Gas & $\begin{array}{c}\text { Distillate } \\
\text { Fuel Oil }\end{array}$ & Gasoline & Natural Gas & $\begin{array}{l}\text { Distillate } \\
\text { Fuel Oil }\end{array}$ & \\
\hline & $1000 \mathrm{ft}^{3} * *$ & $1000 \mathrm{gal}$ & $1000 \mathrm{gal}$ & $1000 \mathrm{ft}^{3 * *}$ & $1000 \mathrm{gal}$ & $1000 \mathrm{ft}^{3} * *$ \\
\hline $\mathrm{CO}_{2 \mathrm{e}}$ & $1.37 \mathrm{E}+02$ & $2.27 \mathrm{E}+04$ & $1.76 \mathrm{E}+04$ & $1.25 \mathrm{E}+02$ & $2.29 \mathrm{E}+04$ & $1.21 \mathrm{E}+02$ \\
\hline $\mathrm{CO}_{2}$ & $1.16 \mathrm{E}+02$ & $2.25 E+04$ & $1.72 E+04$ & $1.22 \mathrm{E}+02$ & $2.28 E+04$ & $1.20 \mathrm{E}+02$ \\
\hline $\mathrm{CH}_{4}$ & 8.38E-01 & $1.20 \mathrm{E}+00$ & $8.31 \mathrm{E}+00$ & $5.26 \mathrm{E}-02$ & $2.58 \mathrm{E}-01$ & 2.30E-03 \\
\hline $\mathrm{N}_{2} \mathrm{O}$ & $3.41 \mathrm{E}-03$ & $6.11 \mathrm{E}-01$ & $5.51 \mathrm{E}-01$ & $4.54 \mathrm{E}-03$ & $6.11 \mathrm{E}-01$ & $2.20 \mathrm{E}-03$ \\
\hline $\mathrm{NO}_{\mathrm{x}}$ & $3.56 \mathrm{E}+00$ & $4.76 \mathrm{E}+02$ & $3.02 \mathrm{E}+02$ & $3.51 \mathrm{E}-01$ & $4.02 \mathrm{E}+01$ & $9.40 \mathrm{E}-02$ \\
\hline $\mathrm{SO}_{\mathrm{x}}$ & $6.32 \mathrm{E}-04$ & $3.24 \mathrm{E}+01$ & $4.18 \mathrm{E}+00$ & $6.32 \mathrm{E}-04$ & $3.24 \mathrm{E}+01$ & $6.00 \mathrm{E}-04$ \\
\hline $\mathrm{CO}$ & $2.29 \mathrm{E}+00$ & $1.26 \mathrm{E}+02$ & $1.22 \mathrm{E}+03$ & $1.75 \mathrm{E}-01$ & $2.66 \mathrm{E}+00$ & $4.00 \mathrm{E}-02$ \\
\hline VOC & $2.06 \mathrm{E}-03$ & $1.22 \mathrm{E}+01$ & $2.56 \mathrm{E}+01$ & $2.06 \mathrm{E}-03$ & $4.08 \mathrm{E}-01$ & $5.50 \mathrm{E}-03$ \\
\hline Lead & $5.00 \mathrm{E}-07$ & $\mathrm{ND}^{\dagger}$ & $\mathrm{ND}^{\dagger}$ & $5.00 \mathrm{E}-07$ & 1.40E-08 & $5.00 \mathrm{E}-07$ \\
\hline Mercury & 2.60E-07 & $\mathrm{ND}^{\dagger}$ & $\mathrm{ND}^{\dagger}$ & 2.60E-07 & 1.20E-09 & $2.60 \mathrm{E}-07$ \\
\hline PM10 & 1.66E-02 & $1.49 \mathrm{E}+01$ & $2.40 \mathrm{E}+00$ & 2.64E-02 & $5.19 \mathrm{E}+00$ & $7.60 \mathrm{E}-03$ \\
\hline
\end{tabular}

data from EPA's AP-42, volume 1, 5th edition, 1995 (EPA 2005b)

** Gas volume at $60^{\circ} \mathrm{F}$ and 14.70 psia.

${ }^{\dagger}$ no data available

Table 11 Emission Factors for On-Site Combustion in Other Equipment ( $k g$ of pollutant per unit of fuel)

\begin{tabular}{|c|c|c|c|c|c|c|}
\hline \multirow{3}{*}{$\begin{array}{l}\text { Pollutant } \\
\text { (kg) }\end{array}$} & \multicolumn{3}{|c|}{ Stationary Reciprocating Engine } & \multicolumn{2}{|c|}{ Small Turbine } & \multirow{2}{*}{$\begin{array}{c}\begin{array}{c}\text { Residential } \\
\text { Furnace * }\end{array} \\
\text { Natural Gas }\end{array}$} \\
\hline & Natural Gas & $\begin{array}{c}\text { Distillate } \\
\text { Fuel Oil }\end{array}$ & Gasoline & Natural Gas & $\begin{array}{c}\text { Distillate } \\
\text { Fuel Oil }\end{array}$ & \\
\hline & $1000 \mathrm{~m}^{3}$ ** & $1000 \mathrm{~L}$ & $1000 \mathrm{~L}$ & $1000 \mathrm{~m}^{3 * *}$ & $1000 \mathrm{~L}$ & $1000 m^{3 * *}$ \\
\hline $\mathrm{CO}_{2 \mathrm{e}}$ & $2.19 \mathrm{E}+00$ & $2.72 \mathrm{E}+03$ & $2.11 \mathrm{E}+03$ & $2.00 \mathrm{E}+00$ & $2.75 E+03$ & $1.93 E+00$ \\
\hline $\mathrm{CO}_{2}$ & $1.86 \mathrm{E}+00$ & $2.70 \mathrm{E}+03$ & $2.07 E+03$ & $1.96 \mathrm{E}+00$ & $2.73 E+03$ & $1.92 \mathrm{E}+00$ \\
\hline $\mathrm{CH}_{4}$ & 1.34E-02 & $1.44 \mathrm{E}-01$ & 9.96E-01 & $8.42 \mathrm{E}-04$ & 3.09E-02 & 3.68E-05 \\
\hline $\mathrm{N}_{2} \mathrm{O}$ & 5.46E-05 & 7.32E-02 & 6.60E-02 & $7.28 \mathrm{E}-05$ & 7.32E-02 & 3.52E-05 \\
\hline $\mathrm{NO}_{x}$ & 5.70E-02 & $5.70 \mathrm{E}+01$ & $3.62 \mathrm{E}+01$ & 5.62E-03 & $4.82 \mathrm{E}+00$ & $1.51 \mathrm{E}-03$ \\
\hline $\mathrm{SO}_{\mathrm{x}}$ & $1.01 \mathrm{E}-05$ & $3.88 \mathrm{E}+00$ & 5.01E-01 & 1.01E-05 & $3.88 \mathrm{E}+00$ & 9.61E-06 \\
\hline $\mathrm{CO}$ & 3.66E-02 & $1.51 \mathrm{E}+01$ & $1.46 \mathrm{E}+02$ & 2.81E-03 & 3.19E-01 & $6.41 \mathrm{E}-04$ \\
\hline VOC & 1.36E-03 & $1.46 \mathrm{E}+00$ & $3.07 \mathrm{E}+00$ & 3.30E-05 & 4.89E-02 & 8.81E-05 \\
\hline Lead & 8.01E-09 & $\mathrm{ND}^{\dagger}$ & $\mathrm{ND}^{\dagger}$ & 8.01E-09 & 1.68E-09 & 8.01E-09 \\
\hline Mercury & 4.16E-09 & $\mathrm{ND}^{\dagger}$ & $\mathrm{ND}^{\dagger}$ & 4.16E-09 & $1.44 \mathrm{E}-10$ & 4.16E-09 \\
\hline PM10 & 2.67E-04 & $1.78 \mathrm{E}+00$ & 2.87E-01 & $4.22 \mathrm{E}-04$ & $6.22 \mathrm{E}-01$ & 1.22E-04 \\
\hline
\end{tabular}

$*$ data from the EPA's AP-42, volume 1, 5th edition, 1995 (EPA 2005B)

** Gas volume at $15.6^{\circ} \mathrm{C}$ and $101325 \mathrm{~Pa}$.

$\dagger^{\dagger}$ no data available 


\section{Section 6 - References}

ANL (2005). Greenhouse Gases, Regulated Emissions, and Energy Use in Transportation. GREET Version 1.6 (draft). www.transportation.anl.gov/software/GREET/index.html. Center for Transportation Research, Chicago, IL: Argonne National Laboratory (accessed July 26, 2005).

EIA (2005a). Energy Information Administration Glossary of Electricity Terms. www.eia.doe.gov/cneaf/electricity/page/glossary.html. Washington, DC: U.S. Department of Energy (accessed July 6, 2005).

EIA (2005b). Energy Information Administration Energy Glossary. www.eia.doe.gov/glossary/glossary main page.htm. Washington, DC: U.S. Department of Energy. (accessed July 6, 2005).

EIA (2006a). Form 906 and Form 920, www.eia.doe.gov/cneaf/electricity/page/eia906 920.html Energy Information Administration. www.eia.doe.gov/. Washington, DC: U.S. Department of Energy (accessed April 17, 2006).

EIA (2006b). Electric Power Annual 2005, Energy Information Administration. www.eia.doe.gov/. Washington, DC: U.S. Department of Energy (accessed January, 2007).

EPA (2002). eGRID 2002. Emissions and Generation Resource Integrated Database. www.epa.gov/cleanenergy/egrid. Washington, DC: U.S. Environmental Protection Agency.

EPA (2005a). Six Common Air Pollutants. www.epa.gov/oar/urbanair/6poll.html. Washington, DC: U.S. Environmental Protection Agency (accessed September 2005).

EPA (2005b). Clearing House for Inventories \& Emissions Factors (Chief). www.epa.gov/ttn/chief/. Washington, DC: U.S. Environmental Protection Agency (accessed July 29, 2005).

EPA (2007). eGRID 2006 version 2.1. Emissions and Generation Resource Integrated Database. www.epa.gov/cleanenergy/egrid. Washington, DC: U.S. Environmental Protection Agency.

IPCC (2001). Climate Change 2001: A Scientific Basis, Intergovernmental Panel on Climate Change; Houghton, J.T.; Ding, Y.; Griggs, D.J.; Noguer, M.; van der Linden, P.J.; Dai, X.; Johnson, C.A.; and Maskell, K. eds. Cambridge, U.K.: Cambridge University Press.

NERC (2007). NERC. www.nerc.com/. Princeton, NJ: North American Eclectic Reliability Council (accessed May 31, 2007).

NREL (2005). U.S. LCI Database. www.nrel.gov/lci. Golden, CO: National Renewable Energy Laboratory (accessed June 6, 2005).

Spath, P. L., Mann, M. K., and Kerr, D. R. (1999). Life Cycle Assessment of Coal-fired Power Production. NREL/TP-570-25119. Golden, CO: National Renewable Energy Laboratory.

Spath, P. L. and Mann, M. K. (2000). Life Cycle Assessment of a Natural Gas Combined-Cycle Power Generation System. NREL/TP-570-27715. Golden, CO: National Renewable Energy Laboratory. 


\section{Appendix A - Derivation of Energy and Emission Factors}

Table A-1 summarizes the data sources used in deriving the energy and emission factors. The EIA uses 34 fuel type codes for reporting electricity generation. Table A-2 shows how these fuel type codes were mapped to the 11 energy groups used in this document based on similar production and combustion effects.

Table A-3 presents a summary of the steps used for calculating the source energy factors. The electricity from pumped hydro storage (HPS) is sometimes reported as a negative number and sometimes as a positive number. When it was positive, it was added to the Hydro electricity total. Negative values were divided and subtracted from the bituminous coal and nuclear totals, which are the most probable base load energy groups.

The emission factors were calculated by following the steps in Table A-4.

\section{Nomenclature}

\begin{tabular}{|c|c|}
\hline$e_{i, k}$ & Combustion emissions per physical quantity of fuel for each pollutant \\
\hline$e_{p_{i, k}}$ & Combustion emissions per physical quantity of fuel for each pollutant \\
\hline $\mathrm{E}_{\mathrm{j}}$ & Total annual electricity generation by region \\
\hline $\mathrm{E}_{\mathrm{pc}_{\mathrm{i}}}$ & Precombustion energy per quantity of fuel type \\
\hline$f_{i, j}$ & Combustion source energy factor by energy type and region \\
\hline $\mathrm{f}_{\mathrm{pc}} \mathrm{i}_{\mathrm{i}, \mathrm{j}}$ & Precombustion source energy factor by energy type and region \\
\hline $\mathrm{F}_{\mathrm{d}_{\mathrm{j}}}$ & Total source energy factor for delivered electricity by region (includes precombustion) \\
\hline $\mathrm{F}_{\mathrm{g}_{\mathrm{j}}}$ & Combustion source energy factor for generated electricity by region \\
\hline $\mathrm{F}_{\mathrm{pc}}$ & Precombustion source energy factor by region \\
\hline $\mathrm{L}_{\mathrm{j}}$ & $\mathrm{T}^{*} \mathrm{D}$ losses by region \\
\hline $\mathrm{M}_{\mathrm{i}, \mathrm{j}}$ & $\begin{array}{l}\text { Physical quantity of fuel used for electricity generation by fuel type and region (adjusted } \\
\text { for useful thermal heat from CHP plants greater than } 1 \mathrm{MW} \text { ) }\end{array}$ \\
\hline $\mathrm{P}_{\mathrm{g}_{\mathrm{k}, \mathrm{j}}}$ & Total emission factor per generated kWh of electricity by pollutant and region \\
\hline $\mathrm{P}_{\mathrm{d}_{\mathrm{k}, \mathrm{j}}}$ & Total emission factor per delivered $\mathrm{kWh}$ of electricity by pollutant and region \\
\hline$S_{i, j}$ & $\begin{array}{l}\text { Energy quantity of fuel used for electricity generation by fuel type and region (adjusted } \\
\text { for useful thermal heat from CHP plants greater than } 1 \mathrm{MW} \text { ) }\end{array}$ \\
\hline$\delta_{i, j}$ & Fraction of electricity generated by energy type and region \\
\hline$\varepsilon_{\mathrm{i}, \mathrm{j}}$ & Adjusted electricity generation totals for HPS by source energy type and region \\
\hline$\rho_{\mathrm{k}, \mathrm{j}}$ & Combustion emission factor per generated $\mathrm{kWh}$ of electricity by pollutant and region \\
\hline$\rho_{\mathrm{pc}_{\mathrm{k}, \mathrm{j}}}$ & Precombustion emission factor per generated $\mathrm{kWh}$ of electricity by pollutant and region \\
\hline$\vartheta_{\mathrm{i}, \mathrm{j}}$ & $\begin{array}{l}\text { Combustion source energy factor for a composite unit of electricity by energy type and } \\
\text { region }\end{array}$ \\
\hline
\end{tabular}

Subscripts:

$\begin{array}{ll}\mathrm{i} & \text { Fuel type index } \\ \mathrm{j} & \text { Region index } \\ \mathrm{k} & \text { Pollutant index } \\ \mathrm{pc} & \text { Precombustion }\end{array}$


Table A-1 Data Sources

\begin{tabular}{|c|c|}
\hline Data Source & Data Used \\
\hline EIA Forms 906/920 for 2004 (EIA 2006a) & $\begin{array}{l}\text { Power plant data for: } \\
\text { Electric fuel consumption by quantity } \\
\text { Electric fuel consumption by energy } \\
\text { Net electricity generation }\end{array}$ \\
\hline EIA Electric Power Annual 2005 (EIA 2006b) & $\begin{array}{l}\mathrm{CO}_{2} \text { emission factors for bituminous, subbituminous, } \\
\text { and lignite coals; natural gas; and residual fuel oil. } \\
\text { Total annual emissions for } \mathrm{CO}_{2}, \mathrm{NOx} \text {, and SOx for } \\
2004 \text {. }\end{array}$ \\
\hline eGRID 2000 (EPA 2002) & $\begin{array}{l}\text { T\&D losses by NERC region } \\
\text { Annual net imported and exported electricity by state }\end{array}$ \\
\hline eGRID 2006 version 2.1 (EPA 2007) & $\begin{array}{l}\mathrm{Hg}, \mathrm{NOx} \text {, and SOx emission factors for bituminous, } \\
\text { subbituminous, and lignite coals; natural gas; and } \\
\text { residual fuel oil. } \\
\text { Total annual emissions for } \mathrm{CO}_{2} \text {, NOx, Sox, and } \mathrm{Hg} \text { for } \\
2004 \text {. }\end{array}$ \\
\hline Electricity Generation LCI data module (NREL 2005) & T\&D losses by NERC region \\
\hline $\begin{array}{l}\text { LCI data modules for Fuels and Energy } \\
\text { Precombustion and Petroleum Refining (NREL 2005) }\end{array}$ & $\begin{array}{l}\text { Precombustion energy and emissions for processing the } \\
\text { fuels per physical quantity of fuel }\end{array}$ \\
\hline $\begin{array}{l}\text { Biomass Combustion in Utility Turbine LCl data } \\
\text { module (NREL 2005) }\end{array}$ & $\begin{array}{l}\text { Precombustion energy for wood } \\
\text { Emissions for wood combustion in utility turbines }\end{array}$ \\
\hline $\begin{array}{l}\text { LCI data modules for Combustion in Utility Boilers for } \\
\text { Bituminous Coal, Lignite Coal, Natural Gas, Residual } \\
\text { Fuel Oil, and Distillate Fuel Oil (NREL 2005) }\end{array}$ & $\begin{array}{l}\text { Emissions for combustion in utility boilers for each fuel } \\
\text { type }\end{array}$ \\
\hline $\begin{array}{l}\mathrm{LCI} \text { data modules for Combustion in Industrial Boilers } \\
\text { for Bituminous Coal and Lignite Coal (NREL 2005) }\end{array}$ & $\begin{array}{l}\text { Emission data for on-site combustion in building } \\
\text { equipment }\end{array}$ \\
\hline GREET 1.6 (ANL 2005) & $\begin{array}{l}\text { Emission data for on-site combustion in building } \\
\text { equipment }\end{array}$ \\
\hline $\begin{array}{l}\text { Clearing House for Inventories \& Emissions Factors } \\
\text { (EPA 2005b) }\end{array}$ & $\begin{array}{l}\text { Emission data for natural gas combustion in a } \\
\text { residential furnace }\end{array}$ \\
\hline Climate Change 2001 (IPCC 2001) & GWP indices \\
\hline
\end{tabular}


Table A-2 Mapping of EIA Fuel Type Codes to Energy Groups

\begin{tabular}{|c|c|c|}
\hline Energy Group & EIA Fuel Type Codes & EIA Fuel Descriptions \\
\hline Bituminous Coal & $\mathrm{BIT}, \mathrm{WC}, \mathrm{SC}$ & $\begin{array}{l}\text { bituminous coal, anthracite coal, coal, waste coal, } \\
\text { coal based synfuel }\end{array}$ \\
\hline Subbituminous Coal & SUB & subbituminous coal \\
\hline Lignite Coal & LIG & lignite coal \\
\hline Natural Gas & NG, BFG, OG, PG & $\begin{array}{l}\text { natural gas, blast furnace gas, other gas, } \\
\text { gaseous propane }\end{array}$ \\
\hline Petroleum Fuels & $\begin{array}{l}\text { DFO, JF, KER, PC, RFO, } \\
\text { WO }\end{array}$ & $\begin{array}{l}\text { distillate fuel oil, jet fuel, kerosene, petroleum } \\
\text { coke, residual fuel oil, waste oil }\end{array}$ \\
\hline Other Fossil Fuel & TDF, OTH, PUR & Tire-derived fuels, other, purchased steam \\
\hline Nuclear & NUC & nuclear \\
\hline Hydro & $\mathrm{HYC}$ & conventional hydro power \\
\hline Renewable Fuels & $\begin{array}{l}\text { AB, MSW, OBS, WDS, OBL, } \\
\text { BLQ, SLW, WDL, LFG, OBG }\end{array}$ & $\begin{array}{l}\text { agricultural crop by-product, municipal solid } \\
\text { waste, other biomass solids, wood solids, other } \\
\text { biomass liquids, black liquor, sludge waste, wood } \\
\text { waste liquids, landfill gas, other biomass gas }\end{array}$ \\
\hline Geothermal & GEO & geothermal \\
\hline Wind & WND & wind \\
\hline Solar (PV) & SUN & PV solar \\
\hline & HPS * & pumped hydro storage \\
\hline
\end{tabular}

* The electricity from HPS was added to the hydro electricity if it was positive and subtracted from the Bit/sub/anth Coal and Nuclear electricity if it was negative. 
Table A-3 Steps for Calculating Electricity Source Energy Factors

\begin{tabular}{|c|c|}
\hline Step & Description \\
\hline 1 & $\begin{array}{l}\text { Starting with the EIA 906/920 } 2004 \text { data (EIA 2006a), fill in missing NERC region names for } \\
\text { imputed data based on the reported state. Use the NERC region that most of the state lies in. The } \\
\text { imputed data represent } 2.3 \% \text { of the total generation. }\end{array}$ \\
\hline 2 & $\begin{array}{l}\text { Sort the data by NERC region and fuel type. Sort this data again by mapping the EIA Fuel Type } \\
\text { codes to the energy groups as shown in Table A-2. Repeat at the state level. }\end{array}$ \\
\hline 3 & $\begin{array}{l}\text { Adjust the electricity generation totals }\left(\varepsilon_{\mathrm{i}, \mathrm{j}}\right) \text { to include the HPS. } \\
\text { Assumptions: Add positive values to the hydro electricity total. Subtract negative values from the } \\
\text { bituminous coal and nuclear totals }\end{array}$ \\
\hline 4 & $\begin{array}{l}\text { Calculate the fraction of electricity generated by energy type and region (see Table 1). } \\
\qquad \delta_{\mathrm{i}, \mathrm{j}}=\frac{\varepsilon_{\mathrm{i}, \mathrm{j}}}{\mathrm{E}_{\mathrm{j}}}\end{array}$ \\
\hline 5 & $\begin{array}{l}\text { Calculate the combustion source energy factors by energy type and region (see Table B-1). } \\
\qquad \mathrm{f}_{\mathrm{i}, \mathrm{j}}=\frac{\mathrm{S}_{\mathrm{i}, \mathrm{j}}}{\varepsilon_{\mathrm{i}, \mathrm{j}}} \\
\text { Assumptions: The source energy factor for hydro, wind, and solar PV is one. }\end{array}$ \\
\hline 6 & $\begin{array}{l}\text { Calculate the combustion source energy factors for a composite unit of electricity by energy type } \\
\text { and region (see Table B-2). } \\
\qquad \vartheta_{\mathrm{i}, \mathrm{j}}=\mathrm{f}_{\mathrm{i}, \mathrm{j}} * \delta_{\mathrm{i}, \mathrm{j}}\end{array}$ \\
\hline 7 & $\begin{array}{l}\text { Calculate the total combustion source energy factors for generated electricity by region. } \\
\qquad \mathrm{F}_{\mathrm{g}_{\mathrm{j}}}=\sum_{\mathrm{i}} \vartheta_{\mathrm{i}, \mathrm{j}}\end{array}$ \\
\hline 8 & $\begin{array}{l}\text { Determine the precombustion source energy factors by energy type and region. The } \\
\text { precombustion energy per quantity of fuel is from the Fuels and Energy Precombustion } \mathrm{LCl} \text { data } \\
\text { module (NREL 2005). } \\
\qquad \mathrm{f}_{\mathrm{pc}_{\mathrm{i}, \mathrm{j}}}=\frac{\mathrm{E}_{\mathrm{pc}_{\mathrm{i}}} \times \mathrm{M}_{\mathrm{i}, \mathrm{j}}}{\mathrm{E}_{\mathrm{j}}}\end{array}$ \\
\hline 9 & $\begin{array}{l}\text { Calculate the total precombustion source energy factors by region (see Table B-2). } \\
\qquad \mathrm{F}_{\mathrm{pc}_{\mathrm{j}}}=\sum_{\mathrm{i}} \mathrm{f}_{\mathrm{pc}_{\mathrm{i}, \mathrm{j}}}\end{array}$ \\
\hline 10 & $\begin{array}{l}\text { Calculate the total fuel factors for delivered energy including the T\&D losses (see Table B-2). The } \\
\text { T\&D losses (see Table 1) are from the Electricity Generation LCI data module (NREL 2005) and } \\
\text { eGRID } 2000 \text { (EPA 2002). } \\
\qquad \mathrm{F}_{\mathrm{d}_{\mathrm{j}}}=\left(\mathrm{F}_{\mathrm{g}_{\mathrm{j}}}+\mathrm{F}_{\mathrm{pc}_{\mathrm{j}}}\right)\left(1+\mathrm{L}_{\mathrm{j}}\right)\end{array}$ \\
\hline
\end{tabular}


Table A-4 Steps for Calculating Emission Factors for Electricity Generation

\begin{tabular}{|c|c|}
\hline Step & Description \\
\hline 1 & $\begin{array}{l}\text { Extract the emission factors for fuel combustion in utility boilers for each pollutant and fuel type } \\
\text { from the appropriate } \mathrm{LCl} \text { data module. Use the } \mathrm{CO}_{2} \text { emission factors for coal, natural gas, and } \\
\text { residual fuel oil from EIA Electric Power Annual (EIA 2006b). Determine the NOx, SOx, } \mathrm{SO}_{2} \text {, and } \\
\mathrm{Hg} \text { emission factors for coal, natural gas, and residual fuel oil from eGRID } 2006 \text { version } 1 \text { (EPA } \\
2006 \text { ). } \\
\text { Assumptions: Emission factors for combustion of residual fuel oil are used for all petroleum fuels. } \\
\text { Emission factors for bituminous coal are used for other fossil fuels. Emissions for electricity } \\
\text { generation from gasification of wood are used for all renewable fuels. }\end{array}$ \\
\hline 2 & $\begin{array}{l}\text { Compile the precombustion emission factors for each pollutant and fuel type from the Fuels and } \\
\text { Energy Precombustion LCl data module. } \\
\text { Assumptions: The emissions for petroleum products were allocated by mass between all of the } \\
\text { petroleum refining coproducts. }\end{array}$ \\
\hline 3 & $\begin{array}{l}\text { Calculate the combustion emissions for each pollutant and region in lb pollutant/kWh generated } \\
\text { electricity. } \\
\qquad \rho_{\mathrm{k}, \mathrm{j}}=\sum_{\mathrm{i}} \mathrm{e}_{\mathrm{i}, \mathrm{k}} \times \mathrm{M}_{\mathrm{i}, \mathrm{j}}\end{array}$ \\
\hline 4 & $\begin{array}{l}\text { Calculate the precombustion emissions for each pollutant and region in lb pollutant/kWh generated } \\
\text { electricity. } \\
\qquad \rho_{\mathrm{pc}_{\mathrm{k}, \mathrm{j}}}=\sum_{\mathrm{i}} \mathrm{e}_{\mathrm{pc}} \mathrm{c}_{\mathrm{i}, \mathrm{k}} \times \mathrm{M}_{\mathrm{i}, \mathrm{j}}\end{array}$ \\
\hline 5 & $\begin{array}{l}\text { Calculate the total emissions for generated electricity for each pollutant and region. } \\
\qquad \mathrm{P}_{\mathrm{g}_{\mathrm{k}, \mathrm{j}}}=\rho_{\mathrm{k}, \mathrm{j}}+\rho_{\mathrm{pc}_{\mathrm{k}, \mathrm{j}}}\end{array}$ \\
\hline 6 & $\begin{array}{l}\text { Calculate the total emissions for generated electricity for each pollutant and region. } \\
\qquad \mathrm{P}_{\mathrm{d}_{\mathrm{k}, \mathrm{j}}}=\mathrm{P}_{\mathrm{g}_{\mathrm{k}, \mathrm{j}}}\left(1+\mathrm{L}_{\mathrm{TD}_{\mathrm{j}}}\right)\end{array}$ \\
\hline
\end{tabular}




\section{Appendix B - Additional Energy and Emission Data}

The source energy factors for generating electricity for each fuel type are listed in Table B-1. These numbers are calculated from the source energy used and electricity generated as reported by the electricity generators in the EIA Forms 906 and 920 (step 5 of Table A-3). The source energy for hydro, wind, and solar are set equal to the electricity generated, which provides a source energy factor of 1.0.

Table B-1 Source Energy Factors by Fuel Type for Generating Electricity (kWh of source energy per kWh of generated electricity)

\begin{tabular}{|l|c|c|c|c|c|c|}
\hline \multicolumn{1}{|c|}{ Energy Group } & National & Eastern & Western & ERCOT & Alaska & Hawaii \\
\hline \hline Bituminous Coal & 2.996 & 2.993 & 3.035 & & & 5.696 \\
\hline Subbituminous Coal & 3.089 & 3.084 & 3.126 & 3.059 & 3.937 & 2.863 \\
\hline Lignite Coal & 3.262 & 3.252 & & 3.272 & & \\
\hline Natural Gas & 2.627 & 2.629 & 2.631 & 2.620 & 3.405 & 3.198 \\
\hline Petroleum Fuels & 3.117 & 3.094 & 3.404 & 4.022 & 3.311 & 3.049 \\
\hline Other Fossil Fuel & 2.465 & 2.377 & 2.859 & 2.140 & & 2.856 \\
\hline Nuclear & 3.075 & 3.075 & 3.083 & 3.060 & & \\
\hline Hydro & 1.000 & 1.000 & 1.000 & 1.000 & 1.000 & 1.000 \\
\hline Renewable Fuels & 4.459 & 4.527 & 3.947 & 6.419 & 2.868 & 5.157 \\
\hline Geothermal & 6.160 & & 6.160 & & & 6.160 \\
\hline Wind & 1.000 & 1.000 & 1.000 & 1.000 & & 1.000 \\
\hline Solar (PV) & 1.000 & & 1.000 & & & \\
\hline
\end{tabular}

The source energy factors for delivered electricity are listed in Table B-2. These factors represent the components by energy type used to generated one kWh of electricity based on annual energy totals. For example, for every $\mathrm{kWh}$ of electricity delivered to the consumer in the United States, the first column lists all the fuel that went into that electricity based on annual totals. The numbers in table were calculated following steps in Table A-3 (the top section from step 6, the precombustion energy from steps 8 and 9 , and the totals from step 10). 
Table B-2 Source Energy Factor Components per Delivered kWh of Electricity for 2004 ( $k W h$ of source energy per kWh of delivered electricity)

\begin{tabular}{|c|c|c|c|c|c|c|}
\hline Energy Group & National & Eastern & Western & ERCOT & Alaska & Hawaii \\
\hline Bituminous Coal & 0.916 & 1.125 & 0.430 & 0.000 & 0.000 & 0.061 \\
\hline Subbituminous Coal & 0.672 & 0.663 & 0.670 & 0.759 & 0.442 & 0.407 \\
\hline Lignite Coal & 0.081 & 0.049 & 0.000 & 0.562 & 0.000 & 0.000 \\
\hline Natural Gas & 0.528 & 0.367 & 0.780 & 1.502 & 2.134 & 0.051 \\
\hline Petroleum Fuels & 0.096 & 0.122 & 0.017 & 0.024 & 0.428 & 2.568 \\
\hline Other Fossil Fuel & 0.005 & 0.005 & 0.008 & 0.005 & 0.000 & 0.007 \\
\hline Nuclear & 0.671 & 0.774 & 0.329 & 0.441 & 0.000 & 0.000 \\
\hline Hydro & 0.074 & 0.037 & 0.267 & 0.003 & 0.259 & 0.009 \\
\hline Renewable Fuels & 0.073 & 0.083 & 0.057 & 0.015 & 0.005 & 0.233 \\
\hline Geothermal & 0.025 & 0.000 & 0.143 & 0.000 & 0.000 & 0.125 \\
\hline Wind & 0.004 & 0.002 & 0.011 & 0.010 & 0.000 & 0.001 \\
\hline Solar (PV) & 0.000 & 0.000 & 0.001 & 0.000 & 0.000 & 0.000 \\
\hline Fossil Precombustion Energy & 0.152 & 0.148 & 0.128 & 0.233 & 0.283 & 0.413 \\
\hline Total Precombustion Energy & 0.168 & 0.166 & 0.140 & 0.253 & 0.300 & 0.455 \\
\hline \multicolumn{7}{|c|}{ Totals without Precombustion Energy } \\
\hline Total Fossil Fuel & 2.299 & 2.332 & 1.905 & 2.851 & 3.004 & 3.094 \\
\hline Total Nonrenewable Energy & 2.970 & 3.106 & 2.234 & 3.292 & 3.004 & 3.094 \\
\hline Total Energy & 3.146 & 3.228 & 2.713 & 3.321 & 3.268 & 3.462 \\
\hline \multicolumn{7}{|c|}{ Totals with Precombustion Energy } \\
\hline Total Fossil Fuel & 2.450 & 2.480 & 2.033 & 3.084 & 3.287 & 3.507 \\
\hline Total Nonrenewable Energy & 3.138 & 3.272 & 2.374 & 3.545 & 3.304 & 3.549 \\
\hline Total Energy & 3.315 & 3.394 & 2.853 & 3.574 & 3.568 & 3.917 \\
\hline
\end{tabular}

Tables B-3 through B-6 show the emission factors per generated $\mathrm{kWh}$ for combustion in utility boilers and precombustion effects in IP and SI units. The emissions are based on the emissions data from the LCI data modules for each fuel type combustion in utility boilers (NREL 2005), and on the fuel totals used for electricity generation as reported on the 2004 EIA Forms 906 and 920 (EIA 2006). These numbers do not include the effects of losses in T\&D of the electricity. 
Table B-3 Combustion Emission Factors for Generated Electricity (Ib of pollutant per kWh of electricity)

\begin{tabular}{|l|c|c|c|c|c|c|}
\hline \multicolumn{1}{|c|}{ Emission (Ib) } & National & Eastern & Western & ERCOT & Alaska & Hawaii \\
\hline $\mathrm{CO}_{2 \mathrm{e}}$ & $1.37 \mathrm{E}+00$ & $1.43 \mathrm{E}+00$ & $1.07 \mathrm{E}+00$ & $1.38 \mathrm{E}+00$ & $1.26 \mathrm{E}+00$ & $1.48 \mathrm{E}+00$ \\
\hline $\mathrm{CO}_{2}$ & $1.36 \mathrm{E}+00$ & $1.42 \mathrm{E}+00$ & $1.06 \mathrm{E}+00$ & $1.37 \mathrm{E}+00$ & $1.25 \mathrm{E}+00$ & $1.47 \mathrm{E}+00$ \\
\hline $\mathrm{CH}_{4}$ & $1.45 \mathrm{E}-05$ & $1.43 \mathrm{E}-05$ & $1.26 \mathrm{E}-05$ & $2.01 \mathrm{E}-05$ & $2.01 \mathrm{E}-05$ & $1.87 \mathrm{E}-05$ \\
\hline $\mathrm{N}_{2} \mathrm{O}$ & $3.25 \mathrm{E}-05$ & $3.39 \mathrm{E}-05$ & $2.62 \mathrm{E}-05$ & $3.27 \mathrm{E}-05$ & $2.46 \mathrm{E}-05$ & $1.44 \mathrm{E}-05$ \\
\hline $\mathrm{NO} \times$ & $2.03 \mathrm{E}-03$ & $2.21 \mathrm{E}-03$ & $1.45 \mathrm{E}-03$ & $1.53 \mathrm{E}-03$ & $1.24 \mathrm{E}-03$ & $2.29 \mathrm{E}-03$ \\
\hline $\mathrm{SO} \times$ & $5.25 \mathrm{E}-03$ & $5.98 \mathrm{E}-03$ & $3.27 \mathrm{E}-03$ & $2.78 \mathrm{E}-03$ & $1.77 \mathrm{E}-03$ & $6.03 \mathrm{E}-03$ \\
\hline $\mathrm{CO}$ & $2.82 \mathrm{E}-04$ & $2.59 \mathrm{E}-04$ & $2.95 \mathrm{E}-04$ & $4.69 \mathrm{E}-04$ & $6.24 \mathrm{E}-04$ & $3.22 \mathrm{E}-04$ \\
\hline $\mathrm{TNMOC}$ & $4.71 \mathrm{E}-05$ & $4.64 \mathrm{E}-05$ & $4.37 \mathrm{E}-05$ & $6.11 \mathrm{E}-05$ & $7.21 \mathrm{E}-05$ & $5.62 \mathrm{E}-05$ \\
\hline Lead & $1.14 \mathrm{E}-07$ & $1.23 \mathrm{E}-07$ & $8.00 \mathrm{E}-08$ & $1.12 \mathrm{E}-07$ & $5.21 \mathrm{E}-08$ & $1.12 \mathrm{E}-07$ \\
\hline Mercury & $2.70 \mathrm{E}-08$ & $2.98 \mathrm{E}-08$ & $1.65 \mathrm{E}-08$ & $2.32 \mathrm{E}-08$ & $3.28 \mathrm{E}-08$ & $1.56 \mathrm{E}-07$ \\
\hline PM10 & $6.96 \mathrm{E}-05$ & $6.98 \mathrm{E}-05$ & $5.42 \mathrm{E}-05$ & $1.00 \mathrm{E}-04$ & $8.13 \mathrm{E}-05$ & $1.21 \mathrm{E}-04$ \\
\hline Solid Waste & $5.20 \mathrm{E}-02$ & $5.40 \mathrm{E}-02$ & $3.43 \mathrm{E}-02$ & $7.07 \mathrm{E}-02$ & $1.55 \mathrm{E}-02$ & $1.28 \mathrm{E}-02$ \\
\hline
\end{tabular}

Table B-4 Combustion Emission Factors for Generated Electricity (kg of pollutant per kWh of electricity)

\begin{tabular}{|l|c|c|c|c|c|c|}
\hline \multicolumn{1}{|c|}{ Emission (kg) } & National & Eastern & Western & ERCOT & Alaska & Hawaii \\
\hline \hline $\mathrm{CO}_{2 \mathrm{e}}$ & $6.20 \mathrm{E}-01$ & $6.50 \mathrm{E}-01$ & $4.86 \mathrm{E}-01$ & $6.24 \mathrm{E}-01$ & $5.72 \mathrm{E}-01$ & $6.69 \mathrm{E}-01$ \\
\hline $\mathrm{CO}_{2}$ & $6.15 \mathrm{E}-01$ & $6.46 \mathrm{E}-01$ & $4.82 \mathrm{E}-01$ & $6.19 \mathrm{E}-01$ & $5.69 \mathrm{E}-01$ & $6.67 \mathrm{E}-01$ \\
\hline $\mathrm{CH}_{4}$ & $6.58 \mathrm{E}-06$ & $6.50 \mathrm{E}-06$ & $5.69 \mathrm{E}-06$ & $9.11 \mathrm{E}-06$ & $9.11 \mathrm{E}-06$ & $8.48 \mathrm{E}-06$ \\
\hline $\mathrm{N}_{2} \mathrm{O}$ & $1.47 \mathrm{E}-05$ & $1.54 \mathrm{E}-05$ & $1.19 \mathrm{E}-05$ & $1.48 \mathrm{E}-05$ & $1.12 \mathrm{E}-05$ & $6.53 \mathrm{E}-06$ \\
\hline $\mathrm{NO} \mathrm{X}_{\mathrm{X}}$ & $9.19 \mathrm{E}-04$ & $1.00 \mathrm{E}-03$ & $6.59 \mathrm{E}-04$ & $6.93 \mathrm{E}-04$ & $5.64 \mathrm{E}-04$ & $1.04 \mathrm{E}-03$ \\
\hline $\mathrm{SO} \mathrm{X}_{\mathrm{NO}}$ & $2.38 \mathrm{E}-03$ & $2.71 \mathrm{E}-03$ & $1.48 \mathrm{E}-03$ & $1.26 \mathrm{E}-03$ & $8.04 \mathrm{E}-04$ & $2.74 \mathrm{E}-03$ \\
\hline $\mathrm{CO}$ & $1.28 \mathrm{E}-04$ & $1.17 \mathrm{E}-04$ & $1.34 \mathrm{E}-04$ & $2.13 \mathrm{E}-04$ & $2.83 \mathrm{E}-04$ & $1.46 \mathrm{E}-04$ \\
\hline $\mathrm{TNMOC}$ & $2.14 \mathrm{E}-05$ & $2.10 \mathrm{E}-05$ & $1.98 \mathrm{E}-05$ & $2.77 \mathrm{E}-05$ & $3.27 \mathrm{E}-05$ & $2.55 \mathrm{E}-05$ \\
\hline Lead & $5.19 \mathrm{E}-08$ & $5.56 \mathrm{E}-08$ & $3.63 \mathrm{E}-08$ & $5.07 \mathrm{E}-08$ & $2.36 \mathrm{E}-08$ & $5.10 \mathrm{E}-08$ \\
\hline Mercury & $1.22 \mathrm{E}-08$ & $1.35 \mathrm{E}-08$ & $7.50 \mathrm{E}-09$ & $1.05 \mathrm{E}-08$ & $1.49 \mathrm{E}-08$ & $7.09 \mathrm{E}-08$ \\
\hline PM10 & $3.16 \mathrm{E}-05$ & $3.16 \mathrm{E}-05$ & $2.46 \mathrm{E}-05$ & $4.55 \mathrm{E}-05$ & $3.69 \mathrm{E}-05$ & $5.50 \mathrm{E}-05$ \\
\hline Solid Waste & $2.36 \mathrm{E}-02$ & $2.45 \mathrm{E}-02$ & $1.56 \mathrm{E}-02$ & $3.21 \mathrm{E}-02$ & $7.03 \mathrm{E}-03$ & $5.81 \mathrm{E}-03$ \\
\hline
\end{tabular}


Table B-5 Precombustion Emission Factors for Generated Electricity (Ib of pollutant per kWh of electricity)

\begin{tabular}{|l|c|c|c|c|c|c|}
\hline \multicolumn{1}{|c|}{ Emission (Ib) } & National & Eastern & Western & ERCOT & Alaska & Hawaii \\
\hline $\mathrm{CO}_{2 \mathrm{e}}$ & $1.54 \mathrm{E}-01$ & $1.52 \mathrm{E}-01$ & $1.38 \mathrm{E}-01$ & $2.09 \mathrm{E}-01$ & $2.50 \mathrm{E}-01$ & $2.77 \mathrm{E}-01$ \\
\hline $\mathrm{CO}_{2}$ & $7.63 \mathrm{E}-02$ & $7.63 \mathrm{E}-02$ & $6.32 \mathrm{E}-02$ & $1.04 \mathrm{E}-01$ & $1.22 \mathrm{E}-01$ & $2.14 \mathrm{E}-01$ \\
\hline $\mathrm{CH}_{4}$ & $3.36 \mathrm{E}-03$ & $3.26 \mathrm{E}-03$ & $3.23 \mathrm{E}-03$ & $4.55 \mathrm{E}-03$ & $5.54 \mathrm{E}-03$ & $2.70 \mathrm{E}-03$ \\
\hline $\mathrm{N}_{2} \mathrm{O}$ & $1.46 \mathrm{E}-06$ & $1.47 \mathrm{E}-06$ & $1.25 \mathrm{E}-06$ & $1.90 \mathrm{E}-06$ & $2.40 \mathrm{E}-06$ & $3.94 \mathrm{E}-06$ \\
\hline $\mathrm{NO} \times$ & $4.80 \mathrm{E}-04$ & $5.24 \mathrm{E}-04$ & $3.45 \mathrm{E}-04$ & $3.68 \mathrm{E}-04$ & $4.81 \mathrm{E}-04$ & $1.68 \mathrm{E}-03$ \\
\hline $\mathrm{SO} \times$ & $2.36 \mathrm{E}-03$ & $1.85 \mathrm{E}-03$ & $3.02 \mathrm{E}-03$ & $5.57 \mathrm{E}-03$ & $8.17 \mathrm{E}-03$ & $2.27 \mathrm{E}-03$ \\
\hline $\mathrm{CO}$ & $4.50 \mathrm{E}-04$ & $5.20 \mathrm{E}-04$ & $2.09 \mathrm{E}-04$ & $3.12 \mathrm{E}-04$ & $1.19 \mathrm{E}-03$ & $6.50 \mathrm{E}-03$ \\
\hline $\mathrm{TNMOC}$ & $1.78 \mathrm{E}-05$ & $1.98 \mathrm{E}-05$ & $1.58 \mathrm{E}-05$ & $2.97 \mathrm{E}-06$ & $2.25 \mathrm{E}-06$ & $4.92 \mathrm{E}-05$ \\
\hline Lead & $4.44 \mathrm{E}-09$ & $4.22 \mathrm{E}-09$ & $2.54 \mathrm{E}-09$ & $1.04 \mathrm{E}-08$ & $3.74 \mathrm{E}-09$ & $8.93 \mathrm{E}-09$ \\
\hline Mercury & $8.13 \mathrm{E}-10$ & $8.50 \mathrm{E}-10$ & $6.16 \mathrm{E}-10$ & $8.96 \mathrm{E}-10$ & $7.86 \mathrm{E}-10$ & $1.53 \mathrm{E}-09$ \\
\hline PM10 & $1.37 \mathrm{E}-05$ & $1.47 \mathrm{E}-05$ & $1.03 \mathrm{E}-05$ & $1.19 \mathrm{E}-05$ & $1.51 \mathrm{E}-05$ & $4.33 \mathrm{E}-05$ \\
\hline Solid Waste & $1.21 \mathrm{E}-01$ & $1.33 \mathrm{E}-01$ & $9.37 \mathrm{E}-02$ & $7.26 \mathrm{E}-02$ & $5.44 \mathrm{E}-02$ & $5.55 \mathrm{E}-02$ \\
\hline
\end{tabular}

Table B-6 Precombustion Emission Factors for Generated Electricity (kg of pollutant per kWh of electricity)

\begin{tabular}{|l|c|c|c|c|c|c|}
\hline \multicolumn{1}{|c|}{ Emission (kg) } & National & Eastern & Western & ERCOT & Alaska & Hawaii \\
\hline \hline $\mathrm{CO}_{2 \mathrm{e}}$ & $6.99 \mathrm{E}-02$ & $6.88 \mathrm{E}-02$ & $6.25 \mathrm{E}-02$ & $9.47 \mathrm{E}-02$ & $1.13 \mathrm{E}-01$ & $1.26 \mathrm{E}-01$ \\
\hline $\mathrm{CO}_{2}$ & $3.46 \mathrm{E}-02$ & $3.46 \mathrm{E}-02$ & $2.87 \mathrm{E}-02$ & $4.70 \mathrm{E}-02$ & $5.53 \mathrm{E}-02$ & $9.71 \mathrm{E}-02$ \\
\hline $\mathrm{CH}_{4}$ & $1.52 \mathrm{E}-03$ & $1.48 \mathrm{E}-03$ & $1.46 \mathrm{E}-03$ & $2.06 \mathrm{E}-03$ & $2.51 \mathrm{E}-03$ & $1.23 \mathrm{E}-03$ \\
\hline $\mathrm{N}_{2} \mathrm{O}$ & $6.64 \mathrm{E}-07$ & $6.65 \mathrm{E}-07$ & $5.66 \mathrm{E}-07$ & $8.60 \mathrm{E}-07$ & $1.09 \mathrm{E}-06$ & $1.79 \mathrm{E}-06$ \\
\hline $\mathrm{NO}$ & $2.18 \mathrm{E}-04$ & $2.38 \mathrm{E}-04$ & $1.56 \mathrm{E}-04$ & $1.67 \mathrm{E}-04$ & $2.18 \mathrm{E}-04$ & $7.60 \mathrm{E}-04$ \\
\hline $\mathrm{SO}$ & $1.07 \mathrm{E}-03$ & $8.38 \mathrm{E}-04$ & $1.37 \mathrm{E}-03$ & $2.53 \mathrm{E}-03$ & $3.71 \mathrm{E}-03$ & $1.03 \mathrm{E}-03$ \\
\hline $\mathrm{CO}$ & $2.04 \mathrm{E}-04$ & $2.36 \mathrm{E}-04$ & $9.49 \mathrm{E}-05$ & $1.42 \mathrm{E}-04$ & $5.42 \mathrm{E}-04$ & $2.95 \mathrm{E}-03$ \\
\hline $\mathrm{TNMOC}$ & $8.05 \mathrm{E}-06$ & $9.00 \mathrm{E}-06$ & $7.17 \mathrm{E}-06$ & $1.35 \mathrm{E}-06$ & $1.02 \mathrm{E}-06$ & $2.23 \mathrm{E}-05$ \\
\hline Lead & $2.01 \mathrm{E}-09$ & $1.91 \mathrm{E}-09$ & $1.15 \mathrm{E}-09$ & $4.74 \mathrm{E}-09$ & $1.70 \mathrm{E}-09$ & $4.05 \mathrm{E}-09$ \\
\hline Mercury & $3.69 \mathrm{E}-10$ & $3.85 \mathrm{E}-10$ & $2.79 \mathrm{E}-10$ & $4.06 \mathrm{E}-10$ & $3.56 \mathrm{E}-10$ & $6.92 \mathrm{E}-10$ \\
\hline PM10 & $6.23 \mathrm{E}-06$ & $6.68 \mathrm{E}-06$ & $4.68 \mathrm{E}-06$ & $5.41 \mathrm{E}-06$ & $6.84 \mathrm{E}-06$ & $1.97 \mathrm{E}-05$ \\
\hline Solid Waste & $5.49 \mathrm{E}-02$ & $6.02 \mathrm{E}-02$ & $4.25 \mathrm{E}-02$ & $3.29 \mathrm{E}-02$ & $2.47 \mathrm{E}-02$ & $2.52 \mathrm{E}-02$ \\
\hline
\end{tabular}

Data on the electricity generation by state are shown in Tables B-7 through B-12. The state data for electricity generation and the fuel used for electricity generation are derived from 2004 EIA Forms 906 and 920 (EIA 2006). The state level data should be used with caution, because several states import electricity from neighboring states, Canada, or Mexico, and the source energy for the imported electricity is not always known. Thirteen states (including the District of Columbia) imported more than $15 \%$ of their electricity in 2000. Table B-7 shows the net imported electricity in GWh (+ for imports and - for exports) and as a percent of the consumed electricity for net importers of electricity and as a percent of the generated electricity for net exporters of electricity. The state net import and export data are from year 2000 data reported in eGRID (EPA 2002).

The percent of electricity generation by fuel type for each state is shown in Table B-8. The source energy factors are in Table B-9 and the emission factors are shown in Tables B-10 and B-11. The ozone season is from May to September, and the $\mathrm{NO}_{\mathrm{X}}$ emissions for this season are shown Table B-12. The ozone season $\mathrm{NO}_{\mathrm{X}}$ emissions do not include precombustion emissions because these emissions do not occur at the power plant, and we are typically only concerned about ozone formation near the power plant. These numbers are for electricity generated within the states and do not include the electricity imported into the state. 
Table B-7 Net Imported Electricity by State for 2000 (EPA 2002)

\begin{tabular}{|c|c|c|c|c|c|c|c|c|c|c|c|c|c|}
\hline & AK & $A L$ & AR & $A Z$ & CA & CO & CT & DC & DE & FL & GA & HI & IA \\
\hline $\begin{array}{l}\text { Net Imported } \\
\text { Electricity (GWh) } \\
\text { (neg. for exported) }\end{array}$ & 0 & $-28,838$ & 1,988 & $-20,072$ & 66,974 & 3,127 & -301 & 10,526 & 5,960 & 23,606 & 8,423 & 0 & 1,662 \\
\hline $\begin{array}{l}\text { Net Imported (+ \% } \\
\text { of consumption) or } \\
\text { Net Exported } \\
\text { (- \% of generation) }\end{array}$ & $0.0 \%$ & $-23.2 \%$ & $4.8 \%$ & $-22.5 \%$ & $26.1 \%$ & $7.2 \%$ & $-0.9 \%$ & $98.8 \%$ & $52.7 \%$ & $12.0 \%$ & $7.0 \%$ & $0.0 \%$ & $4.2 \%$ \\
\hline
\end{tabular}

\begin{tabular}{|c|c|c|c|c|c|c|c|c|c|c|c|c|c|}
\hline & ID & IL & IN & KS & KY & LA & MA & MD & ME & MI & MN & MO & MS \\
\hline $\begin{array}{l}\text { Net Imported } \\
\text { Electricity (GWh) } \\
\text { (neg. for exported) }\end{array}$ & 11,891 & $-25,084$ & $-17,822$ & $-4,350$ & $-5,231$ & -350 & 16,479 & 15,408 & 384 & 10,835 & 13,543 & 3,646 & 11,517 \\
\hline $\begin{array}{l}\text { Net Imported (+ \% } \\
\text { of consumption) or } \\
\text { Net Exported } \\
\text { (- \% of generation) }\end{array}$ & $52.0 \%$ & $-14.1 \%$ & $-13.9 \%$ & $-9.7 \%$ & $-5.6 \%$ & $-0.4 \%$ & $31.8 \%$ & $25.3 \%$ & $3.2 \%$ & $10.3 \%$ & $22.6 \%$ & $5.0 \%$ & $25.3 \%$ \\
\hline
\end{tabular}

\begin{tabular}{|c|c|c|c|c|c|c|c|c|c|c|c|c|c|}
\hline & MT & NC & ND & NE & NH & NJ & NM & NV & NY & $\mathrm{OH}$ & OK & OR & PA \\
\hline $\begin{array}{l}\text { Net Imported } \\
\text { Electricity (GWh) } \\
\text { (neg. for exported) }\end{array}$ & $11,784^{-}$ & 9,883 & $-18,764$ & $-1,864$ & $-3,349$ & 17,454 & $-12,292$ & $-4,807$ & 17,665 & 31,362 & -320 & 3,295 & $-51,727$ \\
\hline $\begin{array}{l}\text { Net Imported (+ \% } \\
\text { of consumption) or } \\
\text { Net Exported } \\
\text { (- \% of generation) }\end{array}$ & $40.9 \%$ & $8.2 \%$ & $-60.0 \%$ & $-6.4 \%$ & $-22.4 \%$ & $24.9 \%$ & $-36.2 \%$ & $-13.5 \%$ & $12.4 \%$ & $18.9 \%$ & $-0.6 \%$ & $6.5 \%$ & $-25.2 \%$ \\
\hline
\end{tabular}

\begin{tabular}{|c|c|c|c|c|c|c|c|c|c|c|c|c|}
\hline & $\mathbf{R} \mathbf{I}$ & Sc & SD & TN & TX & UT & VA & VT & WA & WI & WV & WY \\
\hline $\begin{array}{l}\text { Net Imported } \\
\text { Electricity (GWh) } \\
\text { (neg. for exported) }\end{array}$ & 1,951 & $-6,486$ & -394 & 9,097 & -143 & $-10,236$ & 30,141 & -20 & $-1,067$ & 11,758 & $-56,173$ & $-29,066$ \\
\hline $\begin{array}{l}\text { Net Imported (+ \% } \\
\text { of consumption) or } \\
\text { Net Exported } \\
\text { (- \% of generation) }\end{array}$ & $26.7 \%$ & $-7.0 \%$ & $-4.1 \%$ & $9.5 \%$ & $0.0 \%$ & $-28.0 \%$ & $30.2 \%$ & $-0.3 \%$ & $-1.0 \%$ & $18.0 \%$ & $-60.5 \%$ & $-64.2 \%$ \\
\hline
\end{tabular}


Table B-8 Percent Electricity Generation by Energy Type by State for 2004 (not counting imported electricity)

\begin{tabular}{|c|c|c|c|c|c|c|c|c|c|c|c|c|c|}
\hline Energy Type & AK & $A L$ & AR & $\mathbf{A Z}$ & CA & $\mathrm{CO}$ & CT & DC & $\mathrm{DE}$ & FL & GA & $\mathrm{HI}$ & IA \\
\hline Bit. Coal (\%) & 0.0 & 40.3 & 0.2 & 17.6 & 1.1 & 26.0 & 4.9 & 0.0 & 60.5 & 29.7 & 44.4 & 1.0 & 3.9 \\
\hline Sub. Coal (\%) & 9.9 & 14.2 & 48.6 & 20.4 & 0.0 & 48.6 & 8.2 & 0.0 & 0.0 & 0.0 & 18.2 & 13.1 & 77.7 \\
\hline Lignite Coal (\%) & 0.0 & 0.0 & 0.0 & 0.0 & 0.0 & 0.0 & 0.0 & 0.0 & 0.0 & 0.0 & 0.0 & 0.0 & 0.0 \\
\hline Natural Gas (\%) & 55.5 & 11.8 & 9.7 & 27.0 & 52.5 & 22.4 & 24.8 & 0.0 & 25.6 & 35.1 & 4.9 & 1.5 & 1.9 \\
\hline Pet. Fuels (\%) & 11.5 & 0.2 & 1.0 & 0.0 & 1.2 & 0.0 & 5.3 & 100.0 & 13.9 & 17.1 & 0.7 & 77.4 & 0.3 \\
\hline Other Fossil (\%) & 0.0 & 0.0 & 0.2 & 1.3 & 0.1 & 0.0 & 0.5 & 0.0 & 0.0 & 1.0 & 0.0 & 0.2 & 0.0 \\
\hline Nuclear (\%) & 0.0 & 23.0 & 29.8 & 26.8 & 15.1 & 0.0 & 50.7 & 0.0 & 0.0 & 14.3 & 26.4 & 0.0 & 11.4 \\
\hline Hydro (\%) & 23.0 & 7.7 & 7.1 & 6.7 & 17.5 & 2.5 & 1.4 & 0.0 & 0.0 & 0.1 & 2.9 & 0.8 & 2.2 \\
\hline Ren. Fuel s (\%) & 0.1 & 2.7 & 3.4 & 0.0 & 3.2 & 0.1 & 4.1 & 0.0 & 0.0 & 2.7 & 2.5 & 4.2 & 0.3 \\
\hline Geothermal (\%) & 0.0 & 0.0 & 0.0 & 0.0 & 6.7 & 0.0 & 0.0 & 0.0 & 0.0 & 0.0 & 0.0 & 1.9 & 0.0 \\
\hline Wind (\%) & 0.0 & 0.0 & 0.0 & 0.0 & 2.2 & 0.5 & 0.0 & 0.0 & 0.0 & 0.0 & 0.0 & 0.1 & 2.4 \\
\hline Solar (PV) (\%) & 0.0 & 0.0 & 0.0 & 0.0 & 0.3 & 0.0 & 0.0 & 0.0 & 0.0 & 0.0 & 0.0 & 0.0 & 0.0 \\
\hline Fossil Fuel (\%) & 76.9 & 66.5 & 59.8 & 66.4 & 54.9 & 97.0 & 43.7 & 100.0 & 100.0 & 82.9 & 68.2 & 93.1 & 83.7 \\
\hline $\begin{array}{l}\text { Renewable (\%) } \\
\text { (non Hydro) }\end{array}$ & 0.1 & 2.7 & 3.4 & 0.0 & 12.4 & 0.5 & 4.1 & 0.0 & 0.0 & 2.7 & 2.5 & 6.1 & 2.7 \\
\hline
\end{tabular}

\begin{tabular}{|c|c|c|c|c|c|c|c|c|c|c|c|c|c|}
\hline Energy Type & ID & IL & IN & KS & KY & LA & MA & MD & ME & MI & MN & MO & MS \\
\hline Bit. Coal (\%) & 0.4 & 10.5 & 66.3 & 1.4 & 88.1 & 0.0 & 21.5 & 56.1 & 1.9 & 18.5 & 1.1 & 3.6 & 32.7 \\
\hline Sub. Coal (\%) & 0.5 & 38.7 & 28.2 & 72.5 & 3.0 & 19.3 & 0.0 & 0.0 & 0.0 & 39.2 & 63.9 & 81.9 & 0.0 \\
\hline Lignite Coal (\%) & 0.0 & 0.0 & 0.0 & 0.0 & 0.0 & 4.8 & 0.0 & 0.0 & 0.0 & 0.0 & 0.0 & 0.0 & 7.3 \\
\hline Natural Gas (\%) & 15.7 & 1.9 & 4.4 & 1.8 & 0.6 & 49.9 & 44.2 & 3.1 & 51.4 & 12.7 & 2.9 & 3.3 & 26.7 \\
\hline Pet. Fuels (\%) & 0.0 & 0.4 & 0.4 & 1.8 & 3.8 & 3.9 & 15.8 & 6.3 & 6.9 & 0.8 & 1.5 & 0.2 & 6.4 \\
\hline Other Fossil (\%) & 0.2 & 0.1 & 0.4 & 0.0 & 0.0 & 0.8 & 0.0 & 0.0 & 2.1 & 0.1 & 0.2 & 0.1 & 0.0 \\
\hline Nuclear (\%) & 0.0 & 48.0 & 0.0 & 21.7 & 0.0 & 17.4 & 12.1 & 28.0 & 0.0 & 25.1 & 25.4 & 8.9 & 23.4 \\
\hline Hydro (\%) & 77.9 & 0.1 & 0.3 & 0.0 & 4.0 & 1.1 & 2.1 & 4.8 & 18.0 & 1.3 & 1.4 & 1.8 & 0.0 \\
\hline Ren. Fuel s (\%) & 5.3 & 0.4 & 0.1 & 0.0 & 0.4 & 2.8 & 4.3 & 1.6 & 19.7 & 2.3 & 2.2 & 0.0 & 3.4 \\
\hline Geothermal (\%) & 0.0 & 0.0 & 0.0 & 0.0 & 0.0 & 0.0 & 0.0 & 0.0 & 0.0 & 0.0 & 0.0 & 0.0 & 0.0 \\
\hline Wind $(\%)$ & 0.0 & 0.0 & 0.0 & 0.8 & 0.0 & 0.0 & 0.0 & 0.0 & 0.0 & 0.0 & 1.6 & 0.0 & 0.0 \\
\hline Solar (PV) (\%) & 0.0 & 0.0 & 0.0 & 0.0 & 0.0 & 0.0 & 0.0 & 0.0 & 0.0 & 0.0 & 0.0 & 0.0 & 0.0 \\
\hline Fossil Fuel (\%) & 16.8 & 51.5 & 99.5 & 77.5 & 95.6 & 78.7 & 81.5 & 65.5 & 62.3 & 71.3 & 69.5 & 89.2 & 73.2 \\
\hline $\begin{array}{l}\text { Renewable (\%) } \\
\text { (non Hydro) }\end{array}$ & 5.3 & 0.4 & 0.1 & 0.8 & 0.4 & 2.8 & 4.3 & 1.6 & 19.7 & 2.3 & 3.7 & 0.0 & 3.4 \\
\hline
\end{tabular}


Table B-8 (page 2) Percent Electricity Generation by Energy Type by State for 2004 (not counting imported electricity)

\begin{tabular}{|c|c|c|c|c|c|c|c|c|c|c|c|c|c|}
\hline Energy Type & MT & NC & ND & NE & NH & NJ & NM & NV & NY & $\mathrm{OH}$ & OK & OR & PA \\
\hline Bit. Coal (\%) & 1.1 & 59.8 & 0.0 & 0.0 & 17.1 & 18.3 & 0.1 & 48.5 & 13.8 & 79.4 & 3.7 & 0.0 & 54.4 \\
\hline Sub. Coal (\%) & 62.5 & 0.0 & 1.9 & 63.9 & 0.0 & 0.0 & 88.8 & 0.0 & 2.6 & 7.1 & 51.8 & 6.9 & 0.0 \\
\hline Lignite Coal (\%) & 1.2 & 0.0 & 91.8 & 0.0 & 0.0 & 0.0 & 0.0 & 0.0 & 0.0 & 0.0 & 0.0 & 0.0 & 0.0 \\
\hline Natural Gas (\%) & 0.2 & 2.0 & 0.2 & 0.9 & 22.6 & 28.7 & 9.1 & 43.5 & 19.8 & 1.1 & 38.2 & 26.2 & 4.8 \\
\hline Pet. Fuels (\%) & 1.6 & 0.5 & 0.1 & 0.1 & 8.2 & 2.5 & 0.1 & 0.3 & 15.3 & 0.9 & 0.1 & 0.1 & 1.9 \\
\hline Other Fossil (\%) & 0.0 & 0.2 & 0.0 & 0.0 & 0.0 & 0.0 & 0.0 & 0.0 & 0.0 & 0.0 & 0.0 & 0.0 & 0.0 \\
\hline Nuclear (\%) & 0.0 & 31.7 & 0.0 & 32.0 & 42.6 & 48.1 & 0.0 & 0.0 & 29.1 & 10.8 & 0.0 & 0.0 & 36.0 \\
\hline Hydro (\%) & 33.1 & 4.4 & 5.2 & 2.9 & 5.5 & 0.1 & 0.4 & 4.3 & 17.4 & 0.5 & 4.9 & 64.4 & 1.5 \\
\hline Ren. Fuel s (\%) & 0.2 & 1.4 & 0.0 & 0.1 & 4.0 & 2.3 & 0.0 & 0.0 & 1.9 & 0.3 & 0.4 & 1.1 & 1.3 \\
\hline Geothermal (\%) & 0.0 & 0.0 & 0.0 & 0.0 & 0.0 & 0.0 & 0.0 & 3.4 & 0.0 & 0.0 & 0.0 & 0.0 & 0.0 \\
\hline Wind $(\%)$ & 0.0 & 0.0 & 0.7 & 0.1 & 0.0 & 0.0 & 1.6 & 0.0 & 0.1 & 0.0 & 0.9 & 1.2 & 0.1 \\
\hline Solar (PV) (\%) & 0.0 & 0.0 & 0.0 & 0.0 & 0.0 & 0.0 & 0.0 & 0.0 & 0.0 & 0.0 & 0.0 & 0.0 & 0.0 \\
\hline Fossil Fuel (\%) & 66.7 & 62.5 & 94.1 & 64.9 & 47.9 & 49.5 & 98.0 & 92.3 & 51.6 & 88.5 & 93.8 & 33.3 & 61.2 \\
\hline $\begin{array}{l}\text { Renewable (\%) } \\
\text { (non Hydro) }\end{array}$ & 0.2 & 1.4 & 0.7 & 0.2 & 4.0 & 2.3 & 1.6 & 3.4 & 2.0 & 0.3 & 1.3 & 2.3 & 1.4 \\
\hline
\end{tabular}

\begin{tabular}{|c|c|c|c|c|c|c|c|c|c|c|c|c|c|}
\hline Energy Type & $\mathbf{R} \mathbf{I}$ & sc & SD & TN & TX & UT & VA & VT & WA & WI & WV & WY & Total \\
\hline Bit. Coal (\%) & 0.0 & 39.2 & 0.0 & 47.0 & 0.0 & 95.8 & 44.4 & 0.0 & 0.0 & 10.2 & 97.6 & 0.5 & 27.7 \\
\hline Sub. Coal (\%) & 0.0 & 0.0 & 48.2 & 12.3 & 24.5 & 0.0 & 0.0 & 0.0 & 10.2 & 59.5 & 0.0 & 96.2 & 19.8 \\
\hline Lignite Coal (\%) & 0.0 & 0.0 & 0.0 & 0.0 & 13.6 & 0.0 & 0.0 & 0.0 & 0.0 & 0.0 & 0.0 & 0.0 & 2.2 \\
\hline Natural Gas (\%) & 96.8 & 3.9 & 1.5 & 0.3 & 49.4 & 2.4 & 8.1 & 0.1 & 8.6 & 4.0 & 0.4 & 0.2 & 18.3 \\
\hline Pet. Fuels (\%) & 1.0 & 0.9 & 0.3 & 0.2 & 0.4 & 0.1 & 6.5 & 0.3 & 0.1 & 1.2 & 0.3 & 0.1 & 3.0 \\
\hline Other Fossil (\%) & 0.0 & 0.1 & 0.0 & 0.0 & 0.2 & 0.0 & 0.0 & 0.0 & 0.0 & 0.1 & 0.0 & 0.2 & 0.2 \\
\hline Nuclear (\%) & 0.0 & 51.6 & 0.0 & 29.0 & 10.4 & 0.0 & 35.2 & 70.5 & 8.8 & 19.7 & 0.0 & 0.0 & 19.7 \\
\hline Hydro (\%) & 0.1 & 2.5 & 47.9 & 10.7 & 0.3 & 1.2 & 2.0 & 21.7 & 70.1 & 3.3 & 1.5 & 1.3 & 6.8 \\
\hline Ren. Fuel s (\%) & 2.1 & 1.8 & 0.0 & 0.6 & 0.3 & 0.0 & 3.7 & 7.2 & 1.6 & 1.9 & 0.0 & 0.0 & 1.5 \\
\hline Geothermal (\%) & 0.0 & 0.0 & 0.0 & 0.0 & 0.0 & 0.5 & 0.0 & 0.0 & 0.0 & 0.0 & 0.0 & 0.0 & 0.4 \\
\hline Wind (\%) & 0.0 & 0.0 & 2.1 & 0.0 & 0.8 & 0.0 & 0.0 & 0.2 & 0.7 & 0.2 & 0.2 & 1.4 & 0.4 \\
\hline Solar (PV) (\%) & 0.0 & 0.0 & 0.0 & 0.0 & 0.0 & 0.0 & 0.0 & 0.0 & 0.0 & 0.0 & 0.0 & 0.0 & 0.0 \\
\hline Fossil Fuel (\%) & 97.8 & 44.1 & 50.0 & 59.7 & 88.2 & 98.3 & 59.0 & 0.4 & 18.8 & 75.0 & 98.3 & 97.3 & 71.2 \\
\hline $\begin{array}{l}\text { Renewable (\%) } \\
\text { (non Hydro) }\end{array}$ & 2.1 & 1.8 & 2.1 & 0.6 & 1.1 & 0.5 & 3.7 & 7.4 & 2.3 & 2.0 & 0.2 & 1.4 & 2.2 \\
\hline
\end{tabular}


Table B-9 Source Energy Factors for Delivered Electricity by State for 2004

( $k W h$ of source energy consumed per $k W h$ of delivered electricity)

\begin{tabular}{|c|c|c|c|c|c|c|c|c|c|c|c|c|c|c|c|c|c|}
\hline & AK & $\mathrm{AL}$ & $A R$ & $A Z$ & $\mathrm{CA}$ & $\mathrm{CO}$ & CT & $\mathrm{DC}$ & $\mathrm{DE}$ & $\mathrm{FL}$ & $\mathrm{GA}$ & $\mathrm{HI}$ & IA & ID & IL & IN & KS \\
\hline \multicolumn{18}{|c|}{$\begin{array}{l}\text { Without } \\
\text { Precombustion }\end{array}$} \\
\hline Fossil Fuel & 3.004 & 2.103 & 1.909 & 2.039 & 1.631 & 3.082 & 1.208 & 6.790 & 3.580 & 2.468 & 2.189 & 3.094 & 2.982 & 0.448 & 1.733 & 3.285 & 2.757 \\
\hline Nonrenewable & 3.004 & 2.876 & 2.907 & 2.931 & 2.147 & 3.082 & 2.908 & 6.790 & 3.580 & 2.948 & 3.081 & 3.094 & 3.364 & 0.448 & 3.341 & 3.285 & 3.483 \\
\hline Total & 3.268 & 3.155 & 3.074 & 3.004 & 2.951 & 3.117 & 3.160 & 6.790 & 3.580 & 3.103 & 3.231 & 3.462 & 3.427 & 1.546 & 3.360 & 3.296 & 3.492 \\
\hline \multicolumn{18}{|c|}{ With Precombustion } \\
\hline Fossil Fuel & 3.290 & 2.228 & 2.049 & 2.181 & 1.766 & 3.264 & 1.311 & 7.835 & 3.842 & 2.663 & 2.305 & 3.509 & 3.184 & 0.485 & 1.847 & 3.513 & 3.290 \\
\hline Nonrenewable & 3.308 & 3.016 & 3.068 & 3.089 & 2.291 & 3.283 & 3.028 & 7.938 & 3.862 & 3.161 & 3.215 & 3.551 & 3.593 & 0.488 & 3.477 & 3.535 & 3.308 \\
\hline Total & 3.572 & 3.296 & 3.235 & 3.163 & 3.095 & 3.318 & 3.281 & 7.938 & 3.862 & 3.317 & 3.364 & 3.920 & 3.656 & 1.586 & 3.496 & 3.546 & 3.572 \\
\hline
\end{tabular}

\begin{tabular}{|c|c|c|c|c|c|c|c|c|c|c|c|c|c|c|c|c|c|}
\hline & KY & LA & MA & MD & ME & MI & MN & $\mathrm{MO}$ & MS & MT & NC & ND & NE & $\mathrm{NH}$ & NJ & NM & NV \\
\hline \multicolumn{18}{|l|}{$\begin{array}{l}\text { Without } \\
\text { Precombustion }\end{array}$} \\
\hline Fossil Fuel & 3.202 & 2.452 & 2.352 & 2.161 & 1.662 & 2.297 & 2.362 & 2.966 & 2.384 & 2.389 & 1.984 & 3.337 & 2.207 & 1.449 & 1.591 & 3.311 & 2.802 \\
\hline Nonrenewable & 3.202 & 3.035 & 2.771 & 3.100 & 1.662 & 3.162 & 3.214 & 3.266 & 3.170 & 2.389 & 3.048 & 3.337 & 3.280 & 2.878 & 3.218 & 3.311 & 2.802 \\
\hline Total & 3.277 & 3.128 & 3.029 & 3.248 & 2.693 & 3.285 & 3.348 & 3.288 & 3.273 & 2.776 & 3.172 & 3.402 & 3.317 & 3.187 & 3.333 & 3.333 & 3.079 \\
\hline \multicolumn{18}{|l|}{ With Precombustion } \\
\hline Total Fossil Fuel & 2.952 & 3.351 & 2.658 & 2.556 & 2.335 & 1.817 & 2.433 & 2.521 & 3.166 & 2.568 & 2.565 & 2.074 & 3.640 & 2.360 & 1.565 & 1.706 & 3.531 \\
\hline Total Nonrenewable & 3.705 & 3.367 & 3.259 & 2.993 & 3.294 & 1.829 & 3.314 & 3.396 & 3.492 & 3.374 & 2.586 & 3.153 & 3.678 & 3.458 & 3.012 & 3.349 & 3.556 \\
\hline Total & 3.714 & 3.442 & 3.352 & 3.250 & 3.442 & 2.860 & 3.437 & 3.530 & 3.513 & 3.476 & 2.973 & 3.276 & 3.743 & 3.495 & 3.320 & 3.464 & 3.577 \\
\hline
\end{tabular}

\begin{tabular}{|c|c|c|c|c|c|c|c|c|c|c|c|c|c|c|c|c|c|}
\hline & NY & $\mathrm{OH}$ & OK & OR & PA & RI & SC & SD & TN & $T X$ & UT & VA & VT & WA & WI & WV & WY \\
\hline \multicolumn{18}{|c|}{$\begin{array}{l}\text { Without } \\
\text { Precombustion }\end{array}$} \\
\hline Fossil Fuel & 1.723 & 2.871 & 3.023 & 0.839 & 2.002 & 2.414 & 1.420 & 1.772 & 1.933 & 2.957 & 3.197 & 1.993 & 0.018 & 0.580 & 2.631 & 3.165 & 3.348 \\
\hline Nonrenewable & 2.710 & 3.232 & 3.023 & 0.839 & 3.211 & 2.414 & 3.173 & 1.772 & 2.916 & 3.325 & 3.197 & 3.196 & 2.383 & 0.871 & 3.290 & 3.165 & 3.348 \\
\hline Total & 3.011 & 3.245 & 3.095 & 1.597 & 3.292 & 2.495 & 3.258 & 2.320 & 3.056 & 3.357 & 3.251 & 3.439 & 3.031 & 1.692 & 3.407 & 3.183 & 3.377 \\
\hline \multicolumn{18}{|c|}{ With Precombustion } \\
\hline Fossil Fuel & 1.879 & 3.012 & 3.234 & 0.908 & 2.110 & 2.625 & 1.486 & 1.898 & 2.026 & 3.210 & 3.354 & 2.111 & 0.021 & 0.624 & 2.800 & 3.310 & 3.574 \\
\hline Nonrenewable & 2.885 & 3.390 & 3.254 & 0.913 & 3.336 & 2.635 & 3.255 & 1.913 & 3.025 & 3.600 & 3.370 & 3.333 & 2.400 & 0.922 & 3.483 & 3.324 & 3.602 \\
\hline Total & 3.185 & 3.403 & 3.326 & 1.671 & 3.417 & 2.716 & 3.340 & 2.461 & 3.165 & 3.632 & 3.424 & 3.576 & 3.048 & 1.742 & 3.600 & 3.343 & 3.632 \\
\hline
\end{tabular}


Table B-10 Total Emission Factors for Delivered Electricity by State (Ib of pollutant per kWh of electricity)

\begin{tabular}{|c|c|c|c|c|c|c|c|c|c|c|c|c|c|}
\hline Pollutant (lb) & AK & $A L$ & AR & AZ & CA & co & СТ & DC & DE & FL & GA & HI & IA \\
\hline $\mathrm{CO}_{2 \mathrm{e}}$ & $1.71 \mathrm{E}+00$ & $1.58 \mathrm{E}+00$ & $1.57 \mathrm{E}+00$ & $1.67 \mathrm{E}+00$ & 7.75E-01 & $2.23 E+00$ & $7.29 \mathrm{E}-01$ & $4.26 \mathrm{E}+00$ & $2.43 \mathrm{E}+00$ & $1.49 \mathrm{E}+00$ & $1.62 \mathrm{E}+00$ & $1.91 \mathrm{E}+00$ & $2.41 \mathrm{E}+00$ \\
\hline $\mathrm{CO}_{2}$ & $1.55 \mathrm{E}+00$ & $1.50 \mathrm{E}+00$ & $1.48 \mathrm{E}+00$ & $1.56 \mathrm{E}+00$ & $6.88 \mathrm{E}-01$ & $2.10 \mathrm{E}+00$ & $6.76 \mathrm{E}-01$ & $4.11 \mathrm{E}+00$ & $2.28 \mathrm{E}+00$ & $1.40 \mathrm{E}+00$ & $1.54 \mathrm{E}+00$ & $1.83 \mathrm{E}+00$ & $2.28 \mathrm{E}+00$ \\
\hline $\mathrm{CH}_{4}$ & $6.28 \mathrm{E}-03$ & $3.23 \mathrm{E}-03$ & $3.47 \mathrm{E}-03$ & $4.02 \mathrm{E}-03$ & $3.60 \mathrm{E}-03$ & 4.96E-03 & $2.14 \mathrm{E}-03$ & $6.27 \mathrm{E}-03$ & $5.94 \mathrm{E}-03$ & $3.74 \mathrm{E}-03$ & $2.95 \mathrm{E}-03$ & $2.96 \mathrm{E}-03$ & $4.90 \mathrm{E}-03$ \\
\hline $\mathrm{NO}_{\mathrm{x}}$ & $1.95 \mathrm{E}-03$ & $2.78 \mathrm{E}-03$ & $2.65 \mathrm{E}-03$ & $2.64 \mathrm{E}-03$ & $5.88 \mathrm{E}-04$ & $3.68 \mathrm{E}-03$ & $1.10 \mathrm{E}-03$ & $9.94 \mathrm{E}-03$ & $3.92 \mathrm{E}-03$ & $2.46 \mathrm{E}-03$ & $2.98 \mathrm{E}-03$ & 4.32E-03 & $4.14 \mathrm{E}-03$ \\
\hline $\mathrm{SO}_{\mathrm{x}}$ & 1.12E-02 & $8.24 \mathrm{E}-03$ & $5.13 \mathrm{E}-03$ & $8.86 \mathrm{E}-03$ & $6.42 \mathrm{E}-03$ & $9.64 \mathrm{E}-03$ & $4.23 \mathrm{E}-03$ & $2.15 \mathrm{E}-02$ & $1.53 \mathrm{E}-02$ & $9.44 \mathrm{E}-03$ & $7.73 \mathrm{E}-03$ & $9.04 \mathrm{E}-03$ & $6.75 \mathrm{E}-03$ \\
\hline $\mathrm{CO}$ & $2.05 \mathrm{E}-03$ & 5.33E-04 & $6.44 \mathrm{E}-04$ & 6.16E-04 & 5.36E-04 & 7.78E-04 & 7.89E-04 & 1.96E-02 & $1.85 \mathrm{E}-03$ & $1.80 \mathrm{E}-03$ & $5.20 \mathrm{E}-04$ & 7.43E-03 & $8.19 \mathrm{E}-04$ \\
\hline Mercury & $3.80 \mathrm{E}-08$ & $2.50 \mathrm{E}-08$ & $3.15 \mathrm{E}-08$ & $2.41 \mathrm{E}-08$ & 2.86E-09 & $3.75 \mathrm{E}-08$ & $1.81 \mathrm{E}-08$ & 4.44E-07 & $4.91 \mathrm{E}-08$ & $4.29 \mathrm{E}-08$ & $2.82 \mathrm{E}-08$ & 1.72E-07 & $4.93 \mathrm{E}-08$ \\
\hline PM10 & $1.09 \mathrm{E}-04$ & $7.82 \mathrm{E}-05$ & $9.07 \mathrm{E}-05$ & $8.36 \mathrm{E}-05$ & $4.71 \mathrm{E}-05$ & 1.16E-04 & $4.78 \mathrm{E}-05$ & $4.25 \mathrm{E}-04$ & 1.27E-04 & $8.91 \mathrm{E}-05$ & $7.98 \mathrm{E}-05$ & 1.79E-04 & 1.34E-04 \\
\hline Solid Waste & $7.89 \mathrm{E}-02$ & $1.88 \mathrm{E}-01$ & 2.27E-01 & $1.84 \mathrm{E}-01$ & $1.25 \mathrm{E}-02$ & $2.88 \mathrm{E}-01$ & $5.39 \mathrm{E}-02$ & 7.07E-02 & $2.03 \mathrm{E}-01$ & 1.05E-01 & $2.11 \mathrm{E}-01$ & $7.44 \mathrm{E}-02$ & $3.78 \mathrm{E}-01$ \\
\hline
\end{tabular}

\begin{tabular}{|c|c|c|c|c|c|c|c|c|c|c|c|c|c|}
\hline Pollutant (Ib) & ID & IL & IN & KS & KY & LA & MA & MD & ME & MI & MN & MO & MS \\
\hline $\mathrm{CO}_{2 \mathrm{e}}$ & $2.43 \mathrm{E}-01$ & $1.41 \mathrm{E}+00$ & $2.87 E+00$ & $2.23 \mathrm{E}+00$ & $2.45 \mathrm{E}+00$ & $1.59 \mathrm{E}+00$ & $1.39 \mathrm{E}+00$ & $1.82 \mathrm{E}+00$ & $2.26 \mathrm{E}+00$ & $1.65 \mathrm{E}+00$ & $1.84 \mathrm{E}+00$ & $2.37 \mathrm{E}+00$ & $1.66 \mathrm{E}+00$ \\
\hline $\mathrm{CO}_{2}$ & $2.18 \mathrm{E}-01$ & $1.33 \mathrm{E}+00$ & $2.71 \mathrm{E}+00$ & $2.11 \mathrm{E}+00$ & $2.34 \mathrm{E}+00$ & $1.47 \mathrm{E}+00$ & $1.30 \mathrm{E}+00$ & $1.71 \mathrm{E}+00$ & $2.12 \mathrm{E}+00$ & $1.56 \mathrm{E}+00$ & $1.74 \mathrm{E}+00$ & $2.24 \mathrm{E}+00$ & $1.57 \mathrm{E}+00$ \\
\hline $\mathrm{CH}_{4}$ & $1.03 \mathrm{E}-03$ & $2.77 \mathrm{E}-03$ & 5.93E-03 & 4.56E-03 & $3.88 \mathrm{E}-03$ & 5.02E-03 & 3.94E-03 & 4.02E-03 & 5.43E-03 & $3.57 \mathrm{E}-03$ & $3.82 \mathrm{E}-03$ & 4.85E-03 & 3.72E-03 \\
\hline $\mathrm{NO}_{x}$ & $2.81 \mathrm{E}-04$ & $2.46 \mathrm{E}-03$ & 4.94E-03 & 3.83E-03 & $4.71 \mathrm{E}-03$ & 2.00E-03 & $2.16 \mathrm{E}-03$ & $3.10 \mathrm{E}-03$ & 3.91E-03 & $2.80 \mathrm{E}-03$ & $3.16 \mathrm{E}-03$ & $4.05 \mathrm{E}-03$ & 2.63E-03 \\
\hline $\mathrm{SO}_{\mathrm{x}}$ & $1.92 \mathrm{E}-03$ & $4.76 \mathrm{E}-03$ & 1.49E-02 & $6.24 \mathrm{E}-03$ & $1.34 \mathrm{E}-02$ & $9.05 \mathrm{E}-03$ & $9.18 \mathrm{E}-03$ & $1.11 \mathrm{E}-02$ & 1.42E-02 & $6.74 \mathrm{E}-03$ & $5.20 \mathrm{E}-03$ & $6.70 \mathrm{E}-03$ & $9.86 \mathrm{E}-03$ \\
\hline $\mathrm{CO}$ & $1.53 \mathrm{E}-04$ & 4.85E-04 & $9.42 \mathrm{E}-04$ & $9.19 \mathrm{E}-04$ & 7.25E-04 & $9.84 \mathrm{E}-04$ & $1.91 \mathrm{E}-03$ & 1.19E-03 & 1.46E-03 & $6.38 \mathrm{E}-04$ & 6.67E-04 & $7.98 \mathrm{E}-04$ & $1.18 \mathrm{E}-03$ \\
\hline Mercury & $1.09 \mathrm{E}-09$ & $2.79 \mathrm{E}-08$ & $4.48 \mathrm{E}-08$ & $4.88 \mathrm{E}-08$ & 4.13E-08 & $2.40 \mathrm{E}-08$ & 4.15E-08 & $3.56 \mathrm{E}-08$ & $3.98 \mathrm{E}-08$ & $3.02 \mathrm{E}-08$ & $3.80 \mathrm{E}-08$ & $4.81 \mathrm{E}-08$ & 3.53E-08 \\
\hline PM10 & $1.80 \mathrm{E}-05$ & $7.54 \mathrm{E}-05$ & $1.38 \mathrm{E}-04$ & 1.26E-04 & $1.08 \mathrm{E}-04$ & 1.01E-04 & 8.89E-05 & $9.25 \mathrm{E}-05$ & 1.31E-04 & $8.91 \mathrm{E}-05$ & $1.05 \mathrm{E}-04$ & $1.31 \mathrm{E}-04$ & 1.07E-04 \\
\hline Solid Waste & $7.33 \mathrm{E}-03$ & $2.09 \mathrm{E}-01$ & $3.42 \mathrm{E}-01$ & $3.45 \mathrm{E}-01$ & 3.03E-01 & 1.31E-01 & $7.80 \mathrm{E}-02$ & 1.69E-01 & 1.95E-01 & $2.20 \mathrm{E}-01$ & $2.86 \mathrm{E}-01$ & $3.70 \mathrm{E}-01$ & 1.46E-01 \\
\hline
\end{tabular}


Table B-10 (page 2) Total Emission Factors for Delivered Electricity by State (Ib of pollutant per kWh of electricity)

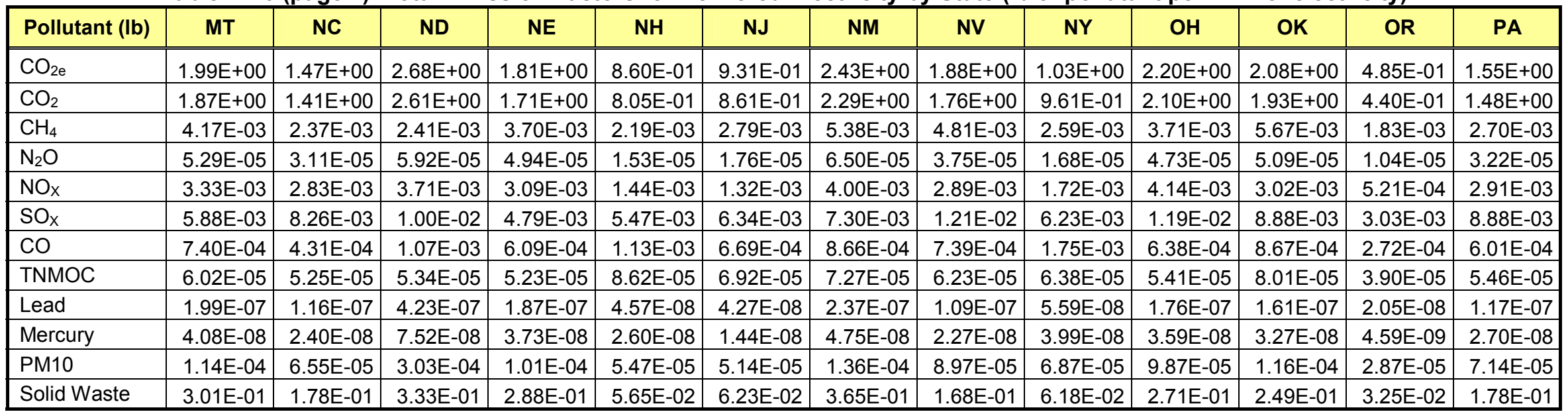

\begin{tabular}{|c|c|c|c|c|c|c|c|c|c|c|c|c|}
\hline Pollutant (lb) & $\mathbf{R I}$ & sc & SD & TN & TX & UT & VA & VT & WA & WI & wV & WY \\
\hline $\mathrm{CO}_{2}$ & $1.04 \mathrm{E}+00$ & $9.57 \mathrm{E}-01$ & $1.36 \mathrm{E}+00$ & $1.40 \mathrm{E}+00$ & $1.85 \mathrm{E}+00$ & $2.51 \mathrm{E}+00$ & $1.33 \mathrm{E}+00$ & $1.78 \mathrm{E}-02$ & $3.82 \mathrm{E}-01$ & $1.92 \mathrm{E}+00$ & $2.31 \mathrm{E}+00$ & $2.52 \mathrm{E}+00$ \\
\hline $\mathrm{CH}_{4}$ & $5.65 \mathrm{E}-03$ & $1.72 \mathrm{E}-03$ & $3.02 \mathrm{E}-03$ & 2.43E-03 & $5.80 \mathrm{E}-03$ & $4.21 \mathrm{E}-03$ & $2.52 \mathrm{E}-03$ & $2.25 \mathrm{E}-05$ & 1.13E-03 & 4.13E-03 & $3.85 \mathrm{E}-03$ & $5.42 \mathrm{E}-03$ \\
\hline $\mathrm{NO}_{\mathrm{x}}$ & $7.91 \mathrm{E}-04$ & $1.90 \mathrm{E}-03$ & $2.45 \mathrm{E}-03$ & $2.77 \mathrm{E}-03$ & $2.42 \mathrm{E}-03$ & $5.00 \mathrm{E}-03$ & $2.67 \mathrm{E}-03$ & $1.38 \mathrm{E}-04$ & $6.13 \mathrm{E}-04$ & $3.51 \mathrm{E}-03$ & $4.62 \mathrm{E}-03$ & $4.58 \mathrm{E}-03$ \\
\hline $\mathrm{SO}_{\mathrm{x}}$ & $9.90 \mathrm{E}-03$ & $5.73 \mathrm{E}-03$ & $3.97 \mathrm{E}-03$ & $7.32 \mathrm{E}-03$ & $1.05 \mathrm{E}-02$ & 1.47E-02 & $8.04 \mathrm{E}-03$ & 1.13E-04 & $1.70 \mathrm{E}-03$ & $6.60 \mathrm{E}-03$ & $1.35 \mathrm{E}-02$ & $7.05 \mathrm{E}-03$ \\
\hline $\mathrm{CO}$ & 8.52E-04 & $3.22 \mathrm{E}-04$ & $5.26 \mathrm{E}-04$ & $4.14 \mathrm{E}-04$ & $9.77 \mathrm{E}-04$ & $6.89 \mathrm{E}-04$ & $9.74 \mathrm{E}-04$ & $5.90 \mathrm{E}-05$ & $1.80 \mathrm{E}-04$ & 7.13E-04 & $6.50 \mathrm{E}-04$ & $9.00 \mathrm{E}-04$ \\
\hline Mercury & $4.09 \mathrm{E}-09$ & $1.62 \mathrm{E}-08$ & $3.01 \mathrm{E}-08$ & $2.50 \mathrm{E}-08$ & $2.96 \mathrm{E}-08$ & $4.15 \mathrm{E}-08$ & $3.24 \mathrm{E}-08$ & 1.03E-09 & $6.62 \mathrm{E}-09$ & $4.01 \mathrm{E}-08$ & $3.87 \mathrm{E}-08$ & $5.54 \mathrm{E}-08$ \\
\hline PM10 & $7.02 \mathrm{E}-05$ & $4.61 \mathrm{E}-05$ & $8.12 \mathrm{E}-05$ & $6.75 \mathrm{E}-05$ & $1.37 \mathrm{E}-04$ & 1.14E-04 & $7.25 \mathrm{E}-05$ & 7.67E-06 & $2.46 \mathrm{E}-05$ & 1.11E-04 & $1.05 \mathrm{E}-04$ & $1.49 \mathrm{E}-04$ \\
\hline Solid Waste & $1.31 \mathrm{E}-02$ & $1.17 \mathrm{E}-01$ & $2.26 \mathrm{E}-01$ & 1.91E-01 & $1.82 \mathrm{E}-01$ & $3.20 \mathrm{E}-01$ & 1.47E-01 & $2.83 \mathrm{E}-04$ & 4.96E-02 & 3.03E-01 & $2.95 \mathrm{E}-01$ & 4.26E-01 \\
\hline
\end{tabular}


Table B-11 Total Emission Factors for Delivered Electricity by State (kg of pollutant per kWh of electricity)

\begin{tabular}{|c|c|c|c|c|c|c|c|c|c|c|c|c|c|}
\hline Poll & $K$ & L & $\mathbf{R}$ & AZ & CA & co & CT & DC & DE & FL & $A$ & HI & IA \\
\hline $\mathrm{CO}_{2 \mathrm{e}}$ & & & 01 & -01 & 01 & $1 \mathrm{E}+00$ & & $93 \mathrm{E}+00$ & $10 \mathrm{E}+00$ & E-01 & $35 \mathrm{E}-01$ & -01 & $1.09 \mathrm{E}+00$ \\
\hline $\mathrm{CO}_{2}$ & & & -01 & OE-01 & 12E-01 & $52 \mathrm{E}-01$ & -01 & $1.86 \mathrm{E}+00$ & $1.03 \mathrm{E}+00$ & $35 \mathrm{E}-01$ & 99E-01 & 32E-01 & $.03 E+00$ \\
\hline $\mathrm{CH}_{4}$ & & & & -03 & $3 \mathrm{E}-03$ & 25E-03 & $72 \mathrm{E}-04$ & $84 \mathrm{E}-03$ & $69 \mathrm{E}-03$ & $0 \mathrm{E}-03$ & $34 \mathrm{E}-03$ & -03 & $22 \mathrm{E}-03$ \\
\hline $\mathrm{NO}$ & & & & & 2.67 & -03 & -04 & -03 & $1.78 \mathrm{E}-03$ & -03 & & -03 & $.88 \mathrm{E}-03$ \\
\hline SO & & 03 & 2.3 & 03 & $2.91 \mathrm{E}-03$ & 4.37 & 1.92E-03 & $9.74 \mathrm{E}-03$ & $95 \mathrm{E}-03$ & -03 & & -03 & $.06 \mathrm{E}-03$ \\
\hline $\mathrm{CO}$ & & 2.42 & 2.9 & & 2.43 & -04 & -04 & 8.9 & $8.38 \mathrm{E}-04$ & -04 & -04 & -03 & $.71 \mathrm{E}-04$ \\
\hline Mercury & $1.72 \mathrm{E}-08$ & 1.13E-08 & $1.43 \mathrm{E}-08$ & $1.09 \mathrm{E}-08$ & 1.30E-09 & 1.70E-08 & $8.19 \mathrm{E}-09$ & $2.01 \mathrm{E}-07$ & $2.23 \mathrm{E}-08$ & $1.95 \mathrm{E}-08$ & $1.28 \mathrm{E}-08$ & $7.79 \mathrm{E}-08$ & 2.24E-08 \\
\hline PM10 & 05 & 3.55 & $=-05$ & -05 & 2.1 & 5.2 & -05 & -04 & -05 & -05 & .05 & -05 & $E-05$ \\
\hline Solid Waste & $3.58 \mathrm{E}-02$ & $8.53 \mathrm{E}-02$ & $1.03 \mathrm{E}-01$ & $8.34 \mathrm{E}-02$ & $5.67 \mathrm{E}-03$ & 1.31E-01 & $2.45 \mathrm{E}-02$ & $3.21 \mathrm{E}-02$ & $9.21 \mathrm{E}-02$ & 4.75E-02 & 9.55E-02 & 3.37E-02 & 1.71E-01 \\
\hline
\end{tabular}

\begin{tabular}{|c|c|c|c|c|c|c|c|c|c|c|c|c|c|}
\hline Pollutant (kg) & ID & IL & IN & KS & KY & LA & MA & MD & ME & MI & MN & MO & MS \\
\hline $\mathrm{CO}_{2 \mathrm{e}}$ & 1.10E-01 & $6.38 \mathrm{E}-01$ & $1.30 \mathrm{E}+00$ & $1.01 \mathrm{E}+00$ & $1.11 \mathrm{E}+00$ & 7.23E-01 & $6.32 \mathrm{E}-01$ & 8.24E-01 & $1.02 \mathrm{E}+00$ & 7.49E-01 & 8.36E-01 & $1.07 \mathrm{E}+00$ & $7.54 \mathrm{E}-01$ \\
\hline $\mathrm{CO}_{2}$ & 9.89E-02 & 6.04E-01 & $1.23 \mathrm{E}+00$ & $9.55 \mathrm{E}-01$ & $1.06 \mathrm{E}+00$ & 6.66E-01 & 5.88E-01 & 7.77E-01 & 9.60E-01 & 7.06E-01 & 7.89E-01 & $1.02 \mathrm{E}+00$ & $7.11 \mathrm{E}-01$ \\
\hline $\mathrm{CH}_{4}$ & $4.67 \mathrm{E}-04$ & $1.26 \mathrm{E}-03$ & $2.69 \mathrm{E}-03$ & $2.07 \mathrm{E}-03$ & $1.76 \mathrm{E}-03$ & $2.28 \mathrm{E}-03$ & $1.79 \mathrm{E}-03$ & $1.82 \mathrm{E}-03$ & $2.46 \mathrm{E}-03$ & $1.62 \mathrm{E}-03$ & $1.73 \mathrm{E}-03$ & $2.20 \mathrm{E}-03$ & $1.69 \mathrm{E}-03$ \\
\hline $\mathrm{NO}_{\mathrm{x}}$ & $1.27 \mathrm{E}-04$ & $1.12 \mathrm{E}-03$ & $2.24 \mathrm{E}-03$ & $1.74 \mathrm{E}-03$ & $2.14 \mathrm{E}-03$ & $9.06 \mathrm{E}-04$ & $9.79 \mathrm{E}-04$ & -03 & 1.77E-03 & $1.27 \mathrm{E}-03$ & $1.44 \mathrm{E}-03$ & $1.84 \mathrm{E}-03$ & $\mathrm{E}-03$ \\
\hline $\mathrm{SO}_{\mathrm{x}}$ & 8.69E-04 & $2.16 \mathrm{E}-03$ & $6.74 \mathrm{E}-03$ & $2.83 \mathrm{E}-03$ & $6.08 \mathrm{E}-03$ & 4.10E-03 & 4.17E-03 & $5.04 \mathrm{E}-03$ & 6.46E-03 & 3.06E-03 & $2.36 \mathrm{E}-03$ & $3.04 \mathrm{E}-03$ & 4.47E-03 \\
\hline $\mathrm{CO}$ & $6.95 \mathrm{E}-05$ & 2.20E-04 & $4.27 \mathrm{E}-04$ & $4.17 \mathrm{E}-04$ & $3.29 E-04$ & 4.46E-04 & $8.67 \mathrm{E}-04$ & $5.40 \mathrm{E}-04$ & 6.61E-04 & 2.89E-04 & E-04 & $3.62 \mathrm{E}-04$ & $5.35 \mathrm{E}-04$ \\
\hline Mercury & $4.93 \mathrm{E}-10$ & 1.27E-08 & $2.03 E-08$ & $2.21 \mathrm{E}-08$ & 1.87E-08 & 1.09E-08 & $1.88 \mathrm{E}-08$ & 1.61E-08 & $1.81 \mathrm{E}-08$ & 1.37E-08 & $1.72 \mathrm{E}-08$ & $2.18 \mathrm{E}-08$ & $1.60 \mathrm{E}-08$ \\
\hline PM10 & $8.18 \mathrm{E}-06$ & $3.42 \mathrm{E}-05$ & $6.26 \mathrm{E}-05$ & $5.70 \mathrm{E}-05$ & 4.91E-05 & 4.57E-05 & 4.03E-05 & 4.19E-05 & 5.94E-05 & 4.04E-05 & 4.76E-05 & $5.95 \mathrm{E}-05$ & 4.85E-05 \\
\hline Solid Waste & 3.33E-03 & $9.49 E-02$ & $1.55 \mathrm{E}-01$ & $1.56 \mathrm{E}-01$ & $1.37 \mathrm{E}-01$ & 5.92E-02 & $3.54 \mathrm{E}-02$ & $7.68 \mathrm{E}-02$ & 8.84E-02 & $9.98 \mathrm{E}-02$ & 1.30E-01 & 1.68E-01 & $6.64 \mathrm{E}-02$ \\
\hline
\end{tabular}


Table B-11 (page 2) Total Emission Factors for Delivered Electricity by State (kg of pollutant per kWh of electricity)

\begin{tabular}{|c|c|c|c|c|c|c|c|c|c|c|c|c|c|}
\hline Poll & MT & $\mathrm{NC}$ & ND & NE & NH & NJ & NM & NV & NY & $\mathrm{OH}$ & OK & OR & PA \\
\hline $0-20$ & & $=-01$ & $22 \mathrm{E}+00$ & 20E-01 & -01 & $22 \mathrm{E}-01$ & $0 \mathrm{E}+00$ & $54 \mathrm{E}-01$ & $E-01$ & $.98 \mathrm{E}-01$ & $9.43 \mathrm{E}-01$ & $2.20 \mathrm{E}-01$ & $.045-U 1$ \\
\hline $\mathrm{CO}_{2}$ & & & & & & & & & & & & & \\
\hline $\mathrm{CH}_{4}$ & & & & & & & & & & 03 & & & $E-03$ \\
\hline $\mathrm{NO}$ & & & & -03 & & 04 & & & & $1.88 \mathrm{E}-03$ & & & -03 \\
\hline SO & & & -03 & $E-03$ & & 2.88 & & -03 & $E-03$ & $5.41 \mathrm{E}-03$ & -03 & -03 & -03 \\
\hline $\mathrm{CO}$ & & -04 & -04 & $E-04$ & 04 & 3.0 & -04 & -04 & $E-04$ & $2.89 \mathrm{E}-04$ & -04 & -04 & $=-04$ \\
\hline Merc & $5 \mathrm{E}-08$ & $1.09 \mathrm{E}-08$ & $3.41 \mathrm{E}-08$ & 1.69E-08 & $1.18 \mathrm{E}-08$ & $6.53 \mathrm{E}-09$ & $2.15 \mathrm{E}-08$ & $1.03 E-08$ & $1.81 \mathrm{E}-08$ & 1.63E-08 & 1.49E-08 & 2.08E-09 & $1.22 \mathrm{E}-08$ \\
\hline PM10 & $5.15 \mathrm{E}-05$ & $2.97 \mathrm{E}-05$ & $1.38 \mathrm{E}-04$ & $4.59 \mathrm{E}-05$ & $2.48 \mathrm{E}-05$ & $2.33 \mathrm{E}-05$ & $6.18 \mathrm{E}-05$ & 4.07E-05 & $3.11 \mathrm{E}-05$ & $4.48 \mathrm{E}-05$ & $5.27 \mathrm{E}-05$ & 1.30E-05 & $3.24 \mathrm{E}-05$ \\
\hline Solid Waste & 1.37E-01 & $8.06 \mathrm{E}-02$ & $1.51 \mathrm{E}-01$ & $1.30 \mathrm{E}-01$ & $2.56 \mathrm{E}-02$ & $2.83 \mathrm{E}-02$ & 1.66E-01 & 7.64E-02 & $2.80 \mathrm{E}-02$ & 1.23E-01 & 1.13E-01 & 1.47E-02 & 8.07E-02 \\
\hline
\end{tabular}

\begin{tabular}{|c|c|c|c|c|c|c|c|c|c|c|c|c|}
\hline Pollutant (kg) & $\mathbf{R} \mathbf{I}$ & sc & SD & TN & TX & UT & VA & VT & WA & WI & wV & WY \\
\hline $\mathrm{CO}_{2}$ & 4.72E-01 & $4.34 \mathrm{E}-01$ & $6.19 \mathrm{E}-01$ & 6.34E-01 & 8.37E-01 & $1.14 \mathrm{E}+00$ & 6.05E-01 & 8.07E-03 & 1.73E-01 & 8.69E-01 & $1.05 \mathrm{E}+00$ & $1.14 \mathrm{E}+00$ \\
\hline $\mathrm{CH}_{4}$ & $2.56 \mathrm{E}-03$ & 7.81E-04 & 1.37E-03 & 1.10E-03 & 2.63E-03 & $1.91 \mathrm{E}-03$ & 1.14E-03 & 1.02E-05 & 5.12E-04 & 1.87E-03 & $1.74 \mathrm{E}-03$ & $2.46 \mathrm{E}-03$ \\
\hline $\mathrm{NO}_{\mathrm{x}}$ & $3.59 \mathrm{E}-04$ & $8.62 \mathrm{E}-04$ & 1.11E-03 & $1.25 \mathrm{E}-03$ & 1.10E-03 & $2.27 \mathrm{E}-03$ & $1.21 \mathrm{E}-03$ & $6.27 \mathrm{E}-05$ & $2.78 \mathrm{E}-04$ & 1.59E-03 & $2.10 \mathrm{E}-03$ & $2.08 \mathrm{E}-03$ \\
\hline $\mathrm{SO}_{\mathrm{x}}$ & $4.49 \mathrm{E}-03$ & $2.60 \mathrm{E}-03$ & $1.80 \mathrm{E}-03$ & $3.32 \mathrm{E}-03$ & $4.77 \mathrm{E}-03$ & 6.67E-03 & $3.65 \mathrm{E}-03$ & $5.14 \mathrm{E}-05$ & 7.73E-04 & 2.99E-03 & $6.13 \mathrm{E}-03$ & $3.20 \mathrm{E}-03$ \\
\hline $\mathrm{CO}$ & 3.86E-04 & 1.46E-04 & 2.39E-04 & $1.88 \mathrm{E}-04$ & 4.43E-04 & 3.13E-04 & 4.42E-04 & $2.68 \mathrm{E}-05$ & $8.16 \mathrm{E}-05$ & $3.23 \mathrm{E}-04$ & $2.95 \mathrm{E}-04$ & $4.08 \mathrm{E}-04$ \\
\hline Mercury & 1.85E-09 & 7.36E-09 & $1.36 \mathrm{E}-08$ & $1.14 \mathrm{E}-08$ & $1.34 \mathrm{E}-08$ & $1.88 \mathrm{E}-08$ & $1.47 \mathrm{E}-08$ & $4.66 \mathrm{E}-10$ & $3.00 \mathrm{E}-09$ & 1.82E-08 & $1.76 \mathrm{E}-08$ & $2.51 \mathrm{E}-08$ \\
\hline PM10 & 3.18E-05 & $2.09 \mathrm{E}-05$ & $3.69 \mathrm{E}-05$ & 3.06E-05 & $6.19 \mathrm{E}-05$ & $5.15 \mathrm{E}-05$ & $3.29 \mathrm{E}-05$ & $3.48 \mathrm{E}-06$ & 1.12E-05 & 5.05E-05 & $4.75 \mathrm{E}-05$ & 6.77E-05 \\
\hline Solid Waste & 5.92E-03 & $5.32 \mathrm{E}-02$ & 1.02E-01 & $8.64 \mathrm{E}-02$ & $8.25 \mathrm{E}-02$ & $1.45 \mathrm{E}-01$ & 6.67E-02 & $1.28 \mathrm{E}-04$ & $2.25 \mathrm{E}-02$ & 1.37E-01 & $1.34 \mathrm{E}-01$ & $1.93 \mathrm{E}-01$ \\
\hline
\end{tabular}


Table B-12 Ozone Season NO Emissions by State for 2004

(mass of $\mathrm{NO}_{x}$ per kWh of delivered electricity without precombustion emissions)

\begin{tabular}{|c|c|c|c|c|c|c|c|c|c|c|c|c|c|c|c|c|c|}
\hline & $A K$ & $\mathrm{AL}$ & $A R$ & $A Z$ & $\mathrm{CA}$ & $\mathrm{CO}$ & CT & $\mathrm{DC}$ & $\mathrm{DE}$ & $\mathrm{FL}$ & $\mathrm{GA}$ & $\mathrm{HI}$ & IA & ID & IL & IN & $\mathrm{KS}$ \\
\hline $\mathrm{Ilb} / \mathrm{kWh}$ & $4.69 \mathrm{E}-3$ & $2.54 \mathrm{E}-3$ & $2.73 \mathrm{E}-3$ & $2.66 \mathrm{E}-3$ & $4.35 \mathrm{E}-4$ & $3.19 \mathrm{E}-3$ & $1.41 \mathrm{E}-3$ & $5.59 \mathrm{E}-3$ & 3.05E-3 & $2.90 \mathrm{E}-3$ & 1.69E-3 & $4.86 \mathrm{E}-3$ & $4.61 \mathrm{E}-3$ & $1.15 \mathrm{E}-4$ & $2.03 \mathrm{E}-3$ & $2.85 \mathrm{E}-3$ & $8.63 \mathrm{E}-3$ \\
\hline $\mathrm{kg} / \mathrm{kWh}$ & $2.13 \mathrm{E}-3$ & $1.15 \mathrm{E}-3$ & $1.24 \mathrm{E}-3$ & $1.21 \mathrm{E}-3$ & $1.97 \mathrm{E}-4$ & $1.45 \mathrm{E}-3$ & $6.38 \mathrm{E}-4$ & $2.54 \mathrm{E}-3$ & $1.39 \mathrm{E}-3$ & $1.31 \mathrm{E}-3$ & $7.66 \mathrm{E}-4$ & $2.21 \mathrm{E}-3$ & $2.09 \mathrm{E}-3$ & $5.22 \mathrm{E}-5$ & $9.22 \mathrm{E}-4$ & $1.29 \mathrm{E}-3$ & $3.92 \mathrm{E}-3$ \\
\hline
\end{tabular}

\begin{tabular}{|c|c|c|c|c|c|c|c|c|c|c|c|c|c|c|c|c|c|}
\hline & KY & LA & MA & MD & $\mathrm{ME}$ & MI & $\mathrm{MN}$ & $\mathrm{MO}$ & MS & MT & $\mathrm{NC}$ & ND & $\mathrm{NE}$ & $\mathrm{NH}$ & NJ & NM & NV \\
\hline $\mathrm{lb} / \mathrm{kWh}$ & $2.24 \mathrm{E}-3$ & $2.38 \mathrm{E}-3$ & $1.44 \mathrm{E}-3$ & $2.76 \mathrm{E}-3$ & $9.82 \mathrm{E}-4$ & $2.82 \mathrm{E}-3$ & $5.09 \mathrm{E}-3$ & $2.75 \mathrm{E}-3$ & $4.27 \mathrm{E}-3$ & $5.59 \mathrm{E}-3$ & $2.28 \mathrm{E}-3$ & $5.60 \mathrm{E}-3$ & $4.69 \mathrm{E}-3$ & $1.74 \mathrm{E}-3$ & $1.84 \mathrm{E}-3$ & $4.91 \mathrm{E}-3$ & $2.67 \mathrm{E}-3$ \\
\hline $\mathrm{kg} / \mathrm{kWh}$ & $1.01 \mathrm{E}-3$ & $1.08 \mathrm{E}-3$ & $6.55 \mathrm{E}-4$ & $1.25 \mathrm{E}-3$ & $4.45 \mathrm{E}-4$ & $1.28 \mathrm{E}-3$ & $2.31 \mathrm{E}-3$ & $1.25 \mathrm{E}-3$ & $1.94 \mathrm{E}-3$ & $2.54 \mathrm{E}-3$ & $1.03 \mathrm{E}-3$ & $2.54 \mathrm{E}-3$ & $2.13 \mathrm{E}-3$ & 7.89E-4 & $8.35 \mathrm{E}-4$ & $2.23 \mathrm{E}-3$ & $1.21 \mathrm{E}-3$ \\
\hline
\end{tabular}

\begin{tabular}{|c|c|c|c|c|c|c|c|c|c|c|c|c|c|c|c|c|c|}
\hline & NY & $\mathrm{OH}$ & OK & OR & PA & $\mathrm{RI}$ & SC & $\mathrm{SD}$ & TN & $T X$ & UT & VA & VT & WA & WI & WV & WY \\
\hline $\mathrm{lb} / \mathrm{kWh}$ & $2.62 \mathrm{E}-3$ & $2.91 \mathrm{E}-3$ & $4.93 \mathrm{E}-4$ & $2.07 \mathrm{E}-3$ & $3.27 \mathrm{E}-4$ & $2.52 \mathrm{E}-3$ & $4.18 \mathrm{E}-3$ & $2.07 \mathrm{E}-3$ & $1.43 \mathrm{E}-3$ & $4.14 \mathrm{E}-3$ & $2.69 \mathrm{E}-3$ & $6.83 \mathrm{E}-4$ & $4.76 \mathrm{E}-4$ & $3.69 \mathrm{E}-3$ & $2.31 \mathrm{E}-3$ & $4.63 \mathrm{E}-3$ & $2.62 \mathrm{E}-3$ \\
\hline $\mathrm{kg} / \mathrm{kWh}$ & $1.19 \mathrm{E}-3$ & $1.32 \mathrm{E}-3$ & 2.24E-4 & $9.38 \mathrm{E}-4$ & $1.48 \mathrm{E}-4$ & $1.14 \mathrm{E}-3$ & $1.90 \mathrm{E}-3$ & $9.37 \mathrm{E}-4$ & $6.48 \mathrm{E}-4$ & $1.88 \mathrm{E}-3$ & $1.22 \mathrm{E}-3$ & $3.10 \mathrm{E}-4$ & $2.16 \mathrm{E}-4$ & $1.67 \mathrm{E}-3$ & $1.05 \mathrm{E}-3$ & $2.10 \mathrm{E}-3$ & $1.19 \mathrm{E}-3$ \\
\hline
\end{tabular}




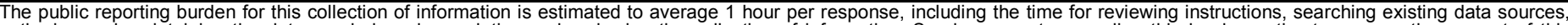

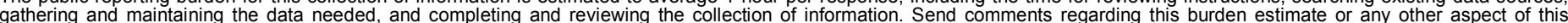

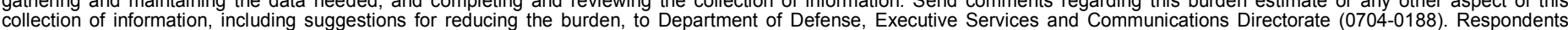

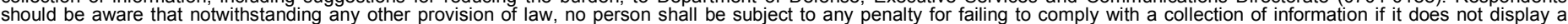

should be aware that notwithstanding

PLEASE DO NOT RETURN YOUR FORM TO THE ABOVE ORGANIZATION.

\begin{tabular}{l|l|l|l} 
1. REPORT DATE $(D D-M M-Y Y Y Y)$ & 2. & REPORT TYPE & 3. DATES COVERED (FrOm - TO)
\end{tabular}

Revised June 2007

Technical Report

4. TITLE AND SUBTITLE
Source Energy and Emission Factors for Energy Use in Buildings

5a. CONTRACT NUMBER

DE-AC36-99-G010337

5b. GRANT NUMBER

5c. PROGRAM ELEMENT NUMBER

6. AUTHOR(S)

M. Deru and P. Torcellini

5d. PROJECT NUMBER

NREL/TP-550-38617

5e. TASK NUMBER

BEC71004

5f. WORK UNIT NUMBER
7. PERFORMING ORGANIZATION NAME(S) AND ADDRESS(ES)

National Renewable Energy Laboratory

1617 Cole Blvd.

Golden, CO 80401-3393
8. PERFORMING ORGANIZATION REPORT NUMBER

NREL/TP-550-38617

9. SPONSORING/MONITORING AGENCY NAME(S) AND ADDRESS(ES)

10. SPONSOR/MONITOR'S ACRONYM(S) NREL

11. SPONSORING/MONITORING AGENCY REPORT NUMBER

12. DISTRIBUTION AVAILABILITY STATEMENT

National Technical Information Service

U.S. Department of Commerce

5285 Port Royal Road

Springfield, VA 22161

\section{SUPPLEMENTARY NOTES}

14. ABSTRACT (Maximum 200 Words)

This document supports the other measurement procedures and all building energy-monitoring projects by providing methods to calculate the source energy and emissions from the energy measured at the building. Energy and emission factors typically account for the conversion inefficiencies at the power plant and the transmission and distribution losses from the power plant to the building. The energy and emission factors provided here also include the precombustion effects, which are the energy and emissions associated with extracting, processing, and delivering the primary fuels to the point of conversion in the electrical power plants or directly in the buildings.

15. SUBJECT TERMS

source energy; source emissions; energy performance metrics

\begin{tabular}{|c|c|c|}
\hline 16. SECURIT & CLASSIFICATI & I OF: \\
\hline $\begin{array}{l}\text { a. REPORT } \\
\text { Unclassified }\end{array}$ & $\begin{array}{l}\text { b. ABSTRACT } \\
\text { Unclassified }\end{array}$ & $\begin{array}{l}\text { c. THIS PAGE } \\
\text { Unclassified }\end{array}$ \\
\hline
\end{tabular}

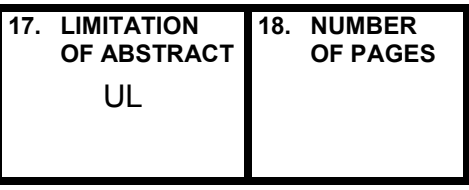

19a. NAME OF RESPONSIBLE PERSON

19b. TELEPHONE NUMBER (Include area code) 